\title{
Numerical method of highly nonlinear and nonautonomous neutral stochastic differential delay equations with Markovian switching
}

\author{
Shuaibin Gao ${ }^{1}$ and Junhao $\mathrm{Hu}^{1 *}$
}

"Correspondence:

junhaohu74@163.com

'College of Mathematics and

Statistics, South-Central University for Nationalities, 430074 Wuhan,

P.R. China

\begin{abstract}
In this paper, we establish a partially truncated Euler-Maruyama scheme for highly nonlinear and nonautonomous neutral stochastic differential delay equations with Markovian switching. We investigate the strong convergence rate and almost sure exponential stability of the numerical solutions under the generalized Khasminskii-type condition.

Keywords: Partially truncated Euler-Maruyama method; Neutral stochastic differential delay equations; Markovian switching; Highly nonlinear and nonautonomous equations
\end{abstract}

\section{Springer}

\section{Introduction}

Stochastic differential equations play an important role in various fields, such as biology, chemistry, and finance [3, 20,27]. In practice, parameters and forms in stochastic systems may change when something unexpected happens. At this point, we can use stochastic differential equations with Markovian switching. Mao and Yuan [24] studied stochastic differential equations with Markovian switching in depth. Many stochastic systems not only depend on the present and past states, but also contain derivatives with delays and the function itself, which can be described by neutral stochastic differential delay equations (NSDDEs) [20]. Kolmanovskii et al. [12] established a fundamental theory for neutral stochastic differential delay equations with Markovian switching (NSDDEwMSs) and discussed some important properties of the solutions.

In many cases the true solutions of the equations cannot be found. So it is very useful to study explicit forms of the numerical solutions. The Euler-Maruyama (EM) method for stochastic differential delay equations with Markovian switching (SDDEwMSs) was investigated in [25] and [37]. Wu and Mao [34] showed the convergence of EM method for neutral stochastic functional differential equations. However, Hutzenthaler et al. [9] showed that $p$ th moments of the EM approximations diverge to infinity for any $p \in[1, \infty)$ when the coefficients grow superlinearly. Many implicit methods were established to estimate the

(c) The Author(s) 2020. This article is licensed under a Creative Commons Attribution 4.0 International License, which permits use, sharing, adaptation, distribution and reproduction in any medium or format, as long as you give appropriate credit to the original author(s) and the source, provide a link to the Creative Commons licence, and indicate if changes were made. The images or other third party material in this article are included in the article's Creative Commons licence, unless indicated otherwise in a credit line to the material. If material is not included in the article's Creative Commons licence and your intended use is not permitted by statutory regulation or exceeds the permitted use, you will need to obtain permission directly from the copyright holder. To view a copy of this licence, visit http://creativecommons.org/licenses/by/4.0/. 
solutions of the equations with superlinearly growing coefficients [2, 4, 8, 11, 26, 30, 32, 33]. Due to the advantages of explicit numerical solutions, such as less computation, plenty of modified EM methods have been studied to approximate the solutions of superlinear stochastic differential equations. The tamed EM scheme was proposed in [10] to estimate the solutions of stochastic differential equations with one-sided Lipschitz drift coefficient and global Lipschitz diffusion coefficient. Sabanis $[28,29]$ developed tamed EM schemes for nonlinear stochastic differential equations. More detail on the other explicit numerical methods can be found in $[1,16,18]$. In addition, Mao initialized the truncated EM method in [21] and obtained the convergence rate in [22]. Then Guo et al. [7] discussed the convergence rate of the truncated EM method for stochastic differential delay equations. The truncated EM method for time-changed nonautonomous stochastic differential equations was shown in [19]. To get the asymptotic behaviors easily, Guo et al. [6] proposed the partially truncated EM method. In [38], the partially truncated EM method for stochastic differential delay equations was proposed. Cong et al. [5] used the partially truncated EM method to get the convergence rate and almost sure exponential stability of highly nonlinear SDDEwMSs. Tan and Yuan in [33] showed the convergence rates of the theta-method for nonlinear neutral stochastic differential delay equations driven by Brownian motion and Poisson jumps, but the stability was not analyzed as time goes to infinity. In [39], the convergence of the EM method for NSDDEwMSs was proved, but the convergence rate was not given. To our best knowledge, there are few papers concerning with numerical solutions of highly nonlinear and nonautonomous NSDDEwMSs. Therefore, in this paper, we give the strong convergence rate of the partially truncated EM method for highly nonlinear and nonautonomous NSDDEwMSs.

Moreover, many scholars are interested in the asymptotic behaviors of the stochastic systems $[3,5,6,20,24,31]$. The almost surely asymptotic stability of NSDDEwMSs was discussed in [23]. Then Li and Mao [15] established LaSalle-type stability theorem for NSDDEwMSs. Liu et al. [17] showed the mean square polynomial stability of the EM method and the backward EM method for stochastic differential equations. The almost sure exponential stability of EM approximations for stochastic differential delay equations was investigated By means of the semimartingale convergence theorem [36]. The exponential mean square stability of the split-step theta method for NSDDEs was investigated in [40]. Lan and Yuan [14] studied the exponential stability of the exact solutions and $\theta$-EM $(1 / 2<\theta \leq 1)$ approximations to NSDDEwMSs. Lan [13] gave the asymptotic mean-square and almost sure exponential stability of the modified truncated EM method for NSDDEs under local Lipschitz condition and nonlinear growth condition. However, there is litthe literature studying the almost sure exponential stability of the partially truncated EM method for highly nonlinear and nonautonomous NSDDEwMSs. The second goal of this paper is to fill this gap.

This paper is organized as follows. We introduce some useful notations and establish the partially truncated EM scheme for NSDDEwMSs in Sect. 2. In Sect. 3, we discuss the strong convergence rate. In Sect. 4, we show the almost sure exponential stability of numerical solutions. Section 5 contains two examples to illustrate that our main result covers a large class of highly nonlinear and nonautonomous NSDDEwMSs.

\section{Mathematical preliminaries}

Unless otherwise specified, we use the following notation. If $A$ is a vector or matrix, its transpose is denoted by $A^{T}$. For $x \in \mathbb{R}^{n}$, let $|x|$ denote its Euclidean norm. If $A$ is a ma- 
trix, denote by $|A|=\sqrt{\operatorname{trace}\left(A^{T} A\right)}$ its trace norm. By $A \leq 0$ and $A<0$ we mean that $A$ is nonpositive and negative definite, respectively. For real numbers $a, b$, we denote $a \wedge b=\min \{a, b\}$ and $a \vee b=\max \{a, b\}$. Let $\lfloor a\rfloor$ be the largest integer that does not exceed $a$. Let $\mathbb{R}_{+}=[0,+\infty)$ and $\tau>0$. By $\mathscr{C}\left([-\tau, 0] ; \mathbb{R}^{n}\right)$ we denote the family of continuous functions $v$ from $[-\tau, 0]$ to $\mathbb{R}^{n}$ with the norm $\|v\|=\sup _{-\tau \leq \tilde{\theta} \leq 0}|v(\tilde{\theta})|$. If $H$ is a set, then $\mathbb{I}_{H}$ denotes its indicator function, that is, $\mathbb{I}_{H}(\omega)=1$ if $\omega \in H$ and $\mathbb{I}_{H}(\omega)=0$ if $\omega \notin H$. Let $C$ stand for a generic positive real constant different in different cases.

Let $\left(\Omega, \mathcal{F},\left\{\mathcal{F}_{t}\right\}_{t \geq 0}, \mathbb{P}\right)$ be a complete probability space with a filtration $\left\{\mathcal{F}_{t}\right\}_{t \geq 0}$ satisfying the usual conditions (i.e., it is increasing and right continuous, and $\mathcal{F}_{0}$ contains all $\mathbb{P}$ null sets). Let $\mathbb{E}$ denote the expectation with respect to $\mathbb{P}$. For $p>0$, let $\mathscr{L}_{\mathcal{F}_{0}}^{p}\left([-\tau, 0] ; \mathbb{R}^{n}\right)$ denote the family of all $\mathcal{F}_{0}$-measurable $\mathscr{C}\left([-\tau, 0] ; \mathbb{R}^{n}\right)$-valued random variables $\xi$ such that $\mathbb{E}\|\xi\|^{p}<\infty$. Let $B(t)=\left(B_{1}(t), \ldots, B_{m}(t)\right)^{T}$ be an $m$-dimensional Brownian motion defined on the probability space.

Let $r(t)(t \geq 0)$ be a right-continuous Markov chain on the probability space taking values in a finite state space $\mathbb{S}=\{1,2, \ldots, N\}$ with generator $\Gamma=\left(\gamma_{i j}\right)_{N \times N}$ given by

$$
\mathbb{P}\{r(t+\Delta)=j \mid r(t)=i\}= \begin{cases}\gamma_{i j}+o(\Delta) & \text { if } i \neq j, \\ 1+\gamma_{i j}+o(\Delta) & \text { if } i=j,\end{cases}
$$

where $\Delta>0$, and $\gamma_{i j}$ is the transition rate from $i$ to $j$ with $\gamma_{i j}>0$ if $i \neq j$, whereas $\gamma_{i i}=$ $-\sum_{j \neq i} \gamma_{i j}$. We suppose that the Markov chain $r$ is independent of the Brownian motion $B$. As is well known [31], almost every sample path of $r$ is a right-continuous step function with finite number of simple jumps in any finite subinterval of $\mathbb{R}_{+}$, that is, there is a sequence of stopping times $0=\tau_{0}<\tau_{1}<\tau_{2}<\cdots<\tau_{k} \rightarrow \infty$ almost surely such that

$$
r(t)=\sum_{k=0}^{\infty} r\left(\tau_{k}\right) \mathbb{I}_{\left[\tau_{k}, \tau_{k+1}\right)}(t),
$$

where $\mathbb{I}$ is the indicator function defined as before. Hence $r$ is constant on each interval $\left[\tau_{k}, \tau_{k+1}\right)$ :

$$
r(t)=r\left(\tau_{k}\right), \quad t \in\left[\tau_{k}, \tau_{k+1}\right), k=0,1,2, \ldots
$$

In this paper, we consider highly nonlinear and nonautonomous neutral stochastic differential delay equations with Markovian switching of the form

$$
\begin{aligned}
& d[x(t)-D(x(t-\tau), r(t))] \\
& \quad=f(t, x(t), x(t-\tau), r(t)) d t+g(t, x(t), x(t-\tau), r(t)) d B(t), \quad t \geq 0,
\end{aligned}
$$

with initial data

$$
x_{0}=\xi \in \mathscr{L}_{\mathcal{F}_{0}}^{p}\left([-\tau, 0] ; \mathbb{R}^{n}\right) \text { and } r(0)=r_{0}
$$

where $r_{0}$ is $\mathbb{S}$-valued $\mathcal{F}_{0}$-measurable random variable. Here $f: \mathbb{R}_{+} \times \mathbb{R}^{n} \times \mathbb{R}^{n} \times \mathbb{S} \rightarrow \mathbb{R}^{n}, g$ : $\mathbb{R}_{+} \times \mathbb{R}^{n} \times \mathbb{R}^{n} \times \mathbb{S} \rightarrow \mathbb{R}^{n \times m}$, and $D: \mathbb{R}^{n} \times \mathbb{S} \rightarrow \mathbb{R}^{n}$. They are all Borel-measurable functions. 
We suppose that the drift and diffusion coefficients can be decomposed as

$$
\begin{aligned}
& f(t, x, y, i)=\tilde{F}(t, x, y, i)+F(t, x, y, i), \\
& g(t, x, y, i)=\tilde{G}(t, x, y, i)+G(t, x, y, i) .
\end{aligned}
$$

To estimate the partially truncated EM method for (2.1), we need the following lemma [24].

Lemma 2.1 Given $\Delta>0$, let $r_{k}^{\Delta}=r(k \Delta)$ for $k \geq 0$. Then $\left\{r_{k}^{\Delta}, k=0,1,2, \ldots\right\}$ is a discrete Markov chain with the one-step transition probability matrix

$$
\mathbb{P}(\Delta)=\left(\mathbb{P}_{i j}(\Delta)\right)_{N \times N}=e^{\Delta \Gamma}
$$

Then we impose two standard necessary hypotheses on the initial data and neutral term.

Assumption 2.2 There exist constants $K_{1}>0$ and $\alpha \in(0,1]$ such that

$$
|\xi(\bar{t})-\xi(\bar{s})| \leq K_{1}|\bar{t}-\bar{s}|^{\alpha}, \quad-\tau \leq \bar{s}<\bar{t} \leq 0 .
$$

Assumption 2.3 (The contractive mapping) $D(0, i)=0$, and there exists a constant $K_{2} \in$ $(0,1)$ such that

$$
|D(x, i)-D(y, i)| \leq K_{2}|x-y|
$$

for all $x, y \in \mathbb{R}^{n}$ and $i \in \mathbb{S}$.

By Assumption 2.3 we have $|D(x, i)| \leq K_{2}|x|$ for all $x \in \mathbb{R}^{n}$ and $i \in \mathbb{S}$.

Since $\gamma_{i j}$ is independent of $x$, the paths of $r$ could be generated before approximating $x$. The discrete Markovian chain $\left\{r_{k}^{\Delta}, k=0,1,2, \ldots\right\}$ can be generated as follows: Compute the one-step transition probability matrix $\mathbb{P}(\Delta)$. Let $r_{0}^{\Delta}=i_{0}$ and generate a random number $\xi_{1}$ uniformly distributed in $[0,1]$. Define

$$
r_{1}^{\Delta}= \begin{cases}i_{1} & \text { if } i_{1} \in \mathbb{S}-\{N\} \text { such that } \sum_{j=1}^{i_{1}-1} \mathbb{P}_{i_{0}, j}(\Delta) \leq \xi_{1}<\sum_{j=1}^{i_{1}} \mathbb{P}_{i_{0}, j}(\Delta), \\ N & \text { if } \sum_{j=1}^{N-1} \mathbb{P}_{i_{0}, j}(\Delta) \leq \xi_{1},\end{cases}
$$

where we set $\sum_{j=1}^{0} \mathbb{P}_{i_{0}, j}(\Delta)=0$ as usual. Then independently generate a new random number $\xi_{2}$ uniformly distributed in $[0,1]$ as well. Define

$$
r_{2}^{\Delta}= \begin{cases}i_{2} & \text { if } i_{2} \in \mathbb{S}-\{N\} \text { such that } \sum_{j=1}^{i_{2}-1} \mathbb{P}_{r_{1}^{\Delta}, j}(\Delta) \leq \xi_{2}<\sum_{j=1}^{i_{2}} \mathbb{P}_{r_{1}^{\Delta}, j}(\Delta), \\ N & \text { if } \sum_{j=1}^{N-1} \mathbb{P}_{r_{1}^{\Delta}, j}(\Delta) \leq \xi_{2} .\end{cases}
$$

Repeating this procedure, we can obtain a trajectory of $\left\{r_{k}^{\Delta}, k=1,2, \ldots\right\}$. The procedure can be applied independently to get more trajectories. After generating the discrete Markov chain $\left\{r_{k}^{\Delta}, k=0,1,2, \ldots\right\}$, we can now define the partially truncated EM approximate solution for NSDDEwMSs (2.1) with initial data (2.2). 
To define the partially truncated EM scheme, we first choose a strictly increasing continuous function $\varphi(w): \mathbb{R}_{+} \rightarrow \mathbb{R}_{+}$such that $\varphi(w) \rightarrow \infty$ as $w \rightarrow \infty$ and

$$
\sup _{0 \leq t \leq T} \sup _{|x| \vee|y| \leq w}(|F(t, x, y, i)| \vee|G(t, x, y, i)|) \leq \varphi(w), \quad \forall w \geq 1
$$

Let $\varphi^{-1}$ denote the inverse function of $\varphi$. Hence $\varphi^{-1}$ is a strictly increasing continuous function from $[\varphi(1), \infty)$ to $\mathbb{R}_{+}$. Then we also choose $K_{0} \geq 1 \vee \varphi(1)$ and a strictly decreasing function $h:(0,1] \rightarrow(0, \infty)$ such that

$$
\lim _{\Delta \rightarrow 0} h(\Delta)=\infty, \quad \Delta^{\frac{1}{4}} h(\Delta) \leq K_{0}, \quad \forall \Delta \in(0,1] .
$$

For a given step size $\Delta \in(0,1]$, define the truncated mapping $\pi_{\Delta}$ from $\mathbb{R}^{n}$ to the closed ball $\left\{x \in \mathbb{R}^{n}:|x| \leq \varphi^{-1}(h(\Delta))\right\}$ by

$$
\pi_{\Delta}(x)=\left(|x| \wedge \varphi^{-1}(h(\Delta))\right) \frac{x}{|x|}
$$

where we let $\frac{x}{|x|}=0$ for $x=0$. Then we can define the truncated functions

$$
F_{\Delta}(t, x, y, i)=F\left(t, \pi_{\Delta}(x), \pi_{\Delta}(y), i\right), \quad G_{\Delta}(t, x, y, i)=G\left(t, \pi_{\Delta}(x), \pi_{\Delta}(y), i\right)
$$

for $x, y \in \mathbb{R}^{n}$. Thus we obtain that

$$
\begin{aligned}
& f_{\Delta}(t, x, y, i)=\tilde{F}(t, x, y, i)+F_{\Delta}(t, x, y, i), \\
& g_{\Delta}(t, x, y, i)=\tilde{G}(t, x, y, i)+G_{\Delta}(t, x, y, i) .
\end{aligned}
$$

Moreover, we can easily get that for any $x, y \in \mathbb{R}^{n}$,

$$
\left|F_{\Delta}(t, x, y, i)\right| \vee\left|G_{\Delta}(t, x, y, i)\right| \leq \varphi\left(\varphi^{-1}(h(\Delta))\right)=h(\Delta) .
$$

Let us now establish our discrete-time truncated EM numerical solutions to approximate the true solution. For some positive integer $M$, we take step size $\Delta=\tau / M$. It is easy to see that $\Delta$ becomes sufficiently small by choosing $M$ sufficiently large. Define $t_{k}=k \Delta$ for $k=-M,-M+1,-M+2, \ldots,-1,0,1,2, \ldots$. Set $X_{\Delta}\left(t_{k}\right)=\xi\left(t_{k}\right)$ for $k=-M,-M+1,-M+$ $2, \ldots,-1,0$ and then form

$$
\begin{aligned}
X_{\Delta}\left(t_{k+1}\right)= & X_{\Delta}\left(t_{k}\right)+D\left(X_{\Delta}\left(t_{k+1-M}\right), r_{k+1}^{\Delta}\right)-D\left(X_{\Delta}\left(t_{k-M}\right), r_{k}^{\Delta}\right) \\
& +f_{\Delta}\left(t_{k}, X_{\Delta}\left(t_{k}\right), X_{\Delta}\left(t_{k-M}\right), r_{k}^{\Delta}\right) \Delta+g_{\Delta}\left(t_{k}, X_{\Delta}\left(t_{k}\right), X_{\Delta}\left(t_{k-M}\right), r_{k}^{\Delta}\right) \Delta B_{k}
\end{aligned}
$$

for $k=0,1,2, \ldots$, where $\Delta B_{k}=B\left(t_{k+1}\right)-B\left(t_{k}\right)$. To form continuous-time step approximations, define

$$
\mu(t)=\sum_{k=0}^{\infty} t_{k} \mathbb{I}_{\left[t_{k}, t_{k+1}\right)}(t), \quad \bar{r}(t)=\sum_{k=0}^{\infty} r_{k}^{\Delta} \mathbb{I}_{\left[t_{k}, t_{k+1}\right)}(t),
$$


where $\mathbb{I}$ is the indicator function. As usual, there are two kinds of continuous-time step approximations. The first one whose sample paths are not continuous is

$$
\bar{x}_{\Delta}(t)=\sum_{k=0}^{\infty} X_{\Delta}\left(t_{k}\right) \mathbb{I}_{\left[t_{k}, t_{k+1}\right)}(t) .
$$

The other one with continuous sample paths is

$$
\begin{aligned}
x_{\Delta}(t)= & \xi(0)+D\left(\bar{x}_{\Delta}(t-\tau), \bar{r}(t)\right)-D\left(\xi(-\tau), r_{0}^{\Delta}\right) \\
& +\int_{0}^{t} f_{\Delta}\left(\mu(s), \bar{x}_{\Delta}(s), \bar{x}_{\Delta}(s-\tau), \bar{r}(s)\right) d s \\
& +\int_{0}^{t} g_{\Delta}\left(\mu(s), \bar{x}_{\Delta}(s), \bar{x}_{\Delta}(s-\tau), \bar{r}(s)\right) d B(s),
\end{aligned}
$$

which is continuous in $t$. Is easy to see that $X_{\Delta}\left(t_{k}\right)=\bar{x}_{\Delta}\left(t_{k}\right)=x_{\Delta}\left(t_{k}\right)$. Namely, they coincide at $t_{k}$.

\section{Strong convergence rate}

In this section, we estimate the strong convergence rate of the partially truncated EM method for (2.1). Now, to achieve this goal, we have to impose the following assumptions on the coefficients.

Assumption 3.1 There exist constants $K_{3}>0$ and $\beta \geq 0$ such that

$$
|\tilde{F}(t, x, y, i)-\tilde{F}(t, \bar{x}, \bar{y}, i)| \vee|\tilde{G}(t, x, y, i)-\tilde{G}(t, \bar{x}, \bar{y}, i)| \leq K_{3}(|x-\bar{x}|+|y-\bar{y}|)
$$

and

$$
\begin{aligned}
& |F(t, x, y, i)-F(t, \bar{x}, \bar{y}, i)| \vee|G(t, x, y, i)-G(t, \bar{x}, \bar{y}, i)| \\
& \quad \leq K_{3}\left(1+|x|^{\beta}+|y|^{\beta}+|\bar{x}|^{\beta}+|\bar{y}|^{\beta}\right)(|x-\bar{x}|+|y-\bar{y}|)
\end{aligned}
$$

for all $t \in[0, T], x, y, \bar{x}, \bar{y} \in \mathbb{R}^{n}$, and $i \in \mathbb{S}$.

By Assumption 3.1 we get that there exists a constant $\bar{K}_{3}>0$ such that

$$
|\tilde{F}(t, x, y, i)| \vee|\tilde{G}(t, x, y, i)| \leq \bar{K}_{3}(1+|x|+|y|)
$$

and

$$
|F(t, x, y, i)| \vee|G(t, x, y, i)| \leq \bar{K}_{3}\left(1+|x|^{\beta+1}+|y|^{\beta+1}\right)
$$

for all $t \in[0, T], x, y \in \mathbb{R}^{n}$, and $i \in \mathbb{S}$, where $\bar{K}_{3}=4 K_{3}+\sup _{t \in[0, T], i \in \mathbb{S}}[\tilde{F}(t, 0,0, i)+\tilde{G}(t, 0,0, i)+$ $F(t, 0,0, i)+G(t, 0,0, i)]$. We also derive from Assumption 3.1 that

$$
\begin{aligned}
& |f(t, x, y, i)-f(t, \bar{x}, \bar{y}, i)| \vee|g(t, x, y, i)-g(t, \bar{x}, \bar{y}, i)| \\
& \quad \leq K_{3}\left(1+|x|^{\beta}+|y|^{\beta}+|\bar{x}|^{\beta}+|\bar{y}|^{\beta}\right)(|x-\bar{x}|+|y-\bar{y}|)
\end{aligned}
$$

for all $t \in[0, T], x, y, \bar{x}, \bar{y} \in \mathbb{R}^{n}$, and $i \in \mathbb{S}$. 
Before stating the next assumption, we introduce functions $\bar{V}_{i}, i=1,2,3$, such that for any $x, y \in \mathbb{R}^{n}$,

$$
0 \leq \bar{V}_{i}(x, y) \leq K_{\bar{V} i}\left(1+|x|^{l_{i}}+|y|^{l_{i}}\right), \quad i=1,2,3,
$$

for some $K_{\bar{V} i}>0$ and $l_{i} \geq 1$. Denote $l_{v}=\max \left\{l_{1}, l_{2}, l_{3}\right\}$.

Assumption 3.2 There exist constants $K_{4}>0$ and $\bar{q}>2$ such that

$$
\begin{aligned}
(x-D(y, i)-\bar{x}+D(\bar{y}, i))^{T}(F(t, x, y, i)-F(t, \bar{x}, \bar{y}, i)) \\
\quad+\frac{\bar{q}-1}{2}|G(t, x, y, i)-G(t, \bar{x}, \bar{y}, i)|^{2} \leq K_{4}|x-\bar{x}|^{2}+\left|\bar{V}_{1}(y, \bar{y})\right||y-\bar{y}|^{2}
\end{aligned}
$$

for all $t \in[0, T], x, y, \bar{x}, \bar{y} \in \mathbb{R}^{n}$, and $i \in \mathbb{S}$.

By Assumption 3.2 we obtain that for any $q \in(2, \bar{q})$,

$$
\begin{aligned}
(x- & D(y, i)-\bar{x}+D(\bar{y}, i))^{T}(f(t, x, y, i)-f(t, \bar{x}, \bar{y}, i)) \\
& +\frac{q-1}{2}|g(t, x, y, i)-g(t, \bar{x}, \bar{y}, i)|^{2} \\
\leq & \left(\bar{K}_{4}+K_{4}\right)|x-\bar{x}|^{2}+\left(\bar{K}_{4}+\left|\bar{V}_{1}(y, \bar{y})\right|\right)|y-\bar{y}|^{2},
\end{aligned}
$$

where $\bar{K}_{4}=2 K_{3}+\frac{K_{3}^{2}(q-1)(\bar{q}-1)}{\bar{q}-q}$. The proof is trivial, so we omit it.

Assumption 3.3 There exist constants $K_{5}>0$ and $\bar{p}>\bar{q}$ such that

$$
\begin{aligned}
(x & -D(y, i))^{T} F(t, x, y, i)+\frac{\bar{p}-1}{2}|G(t, x, y, i)|^{2} \\
& \leq K_{5}\left(1+|x|^{2}\right)+\left|\bar{V}_{2}(y, 0)\right||y|^{2}
\end{aligned}
$$

for all $t \in[0, T], x, y \in \mathbb{R}^{n}$, and $i \in \mathbb{S}$.

By Assumption 3.3 we derive that for any $p \in[2, \bar{p})$,

$$
\begin{aligned}
(x- & D(y, i))^{T} f(t, x, y, i)+\frac{p-1}{2}|g(t, x, y, i)|^{2} \\
\leq & \left(\bar{K}_{5}+K_{5}\right)\left(1+|x|^{2}\right)+\left(\bar{K}_{5}+\left|\bar{V}_{2}(y, 0)\right|\right)|y|^{2},
\end{aligned}
$$

where $\bar{K}_{5}=3 \bar{K}_{3}+\frac{3 \bar{K}_{3}^{2}(p-1)(\bar{p}-1)}{2(\bar{p}-p)}$.

Assumption 3.4 There exist constants $K_{6}>0, K_{7}>0, \theta \in(0,1]$, and $\sigma \in(0,1]$ such that

$$
\begin{aligned}
& \left|f\left(t_{1}, x, y, i\right)-f\left(t_{2}, x, y, i\right)\right| \leq K_{6}\left(1+|x|^{\beta+1}+|y|^{\beta+1}\right)\left|t_{1}-t_{2}\right|^{\theta}, \\
& \left|g\left(t_{1}, x, y, i\right)-g\left(t_{2}, x, y, i\right)\right| \leq K_{7}\left(1+|x|^{\beta+1}+|y|^{\beta+1}\right)\left|t_{1}-t_{2}\right|^{\sigma}
\end{aligned}
$$

for all $t_{1}, t_{2} \in[0, T], x, y \in \mathbb{R}^{n}$, and $i \in \mathbb{S}$, where $\beta$ is as in Assumption 3.1. 
The following lemma gives that the $p$-moment of the true solution is bounded. This lemma can be proved similarly to the proof of Theorem 2.4 presented in [12] by means of the technique used in Theorem 2.1 of [35].

Lemma 3.5 Let Assumptions 3.1 and 3.3 hold. Then neutral stochastic differential delay equations with Markovian switching (2.1) with initial data (2.2) has a unique solution $x(t)$ on $t \geq-\tau$. In addition, this solution has the property that

$$
\sup _{-\tau \leq t \leq T} \mathbb{E}|x(t)|^{p}<\infty, \quad \forall T>0
$$

To get the strong convergence rate, we impose another assumption.

Assumption 3.6 There exist constants $K_{8}>0$ and $\bar{p}>\bar{q}$ such that

$$
\begin{aligned}
(x- & D(y, i))^{T} F_{\Delta}(t, x, y, i)+\frac{\bar{p}-1}{2}\left|G_{\Delta}(t, x, y, i)\right|^{2} \\
& \leq K_{8}\left(1+|x|^{2}\right)+\left|\bar{V}_{3}(y, 0)\right||y|^{2}
\end{aligned}
$$

for all $t \in[0, T], x, y \in \mathbb{R}^{n}$, and $i \in \mathbb{S}$.

By Assumption 3.6 we can show that for any $p \in[2, \bar{p})$,

$$
\begin{aligned}
(x- & D(y, i))^{T} f_{\Delta}(t, x, y, i)+\frac{p-1}{2}\left|g_{\Delta}(t, x, y, i)\right|^{2} \\
\leq & \left(\bar{K}_{8}+K_{8}\right)\left(1+|x|^{2}\right)+\left(\bar{K}_{8}+\left|\bar{V}_{3}(y, 0)\right|\right)|y|^{2},
\end{aligned}
$$

where $\bar{K}_{8}=3 \bar{K}_{3}+\frac{3 \bar{K}_{3}^{2}(p-1)(\bar{p}-1)}{2(\bar{p}-p)}$.

Remark 3.7 When $D(\cdot, \cdot)=0$, we can derive that for any functions satisfying Assumption 3.3,

$$
x^{T} F_{\Delta}(t, x, y, i)+\frac{\bar{p}-1}{2}\left|G_{\Delta}(t, x, y, i)\right|^{2} \leq \tilde{K}_{8}\left(1+|x|^{2}\right)+\left|\bar{V}_{2}(y, 0)\right|^{2}|y|^{2}
$$

for all $t \in[0, T], x, y \in \mathbb{R}^{n}$, and $i \in \mathbb{S}$, where $\tilde{K}_{8}=2 K_{5}\left(\left[1 / \varphi^{-1}(h(1))\right] \vee 1\right)$. In other words, Assumption 3.6 can be eliminated if there is no neutral term.

Remark 3.8 In fact, there are plenty of functions such that $D(y, i), F(t, x, y, i)$, and $G(t, x, y, i)$ satisfy Assumption 3.3 and the corresponding $F_{\Delta}(t, x, y, i)$ and $G_{\Delta}(t, x, y, i)$ satisfy Assumption 3.6. For example, when $i=1$, define $D(y, 1)=-\frac{1}{6} y, f(t, x, y, 1)=-2 y^{3}+(t(1-t))^{\frac{1}{3}} y-$ $10 x+2 y, g(t, x, y, 1)=(t(1-t))^{\frac{1}{3}}|y|^{\frac{3}{2}}$ for $t \in[0,1]$ and $x, y \in \mathbb{R}^{1}$. Thus $F(t, x, y, 1)=-2 y^{3}$ and $G(t, x, y, 1)=(t(1-t))^{\frac{1}{3}}|y|^{\frac{3}{2}}$. We can easily prove that Assumptions 3.3 and 3.6 are satisfied. A detailed proof is presented in Sect. 5 .

Lemma 3.9 Let Assumptions 2.3, 3.1, and 3.6 hold. Then for any $p \in[2, \bar{p})$, we have

$$
\sup _{0<\Delta \leq 1} \sup _{0 \leq t \leq T} \mathbb{E}\left|x_{\Delta}(t)\right|^{p} \leq C, \quad \forall T>0
$$


Proof For any $\Delta \in(0,1]$ and $t \in[0, T]$, by Itô's formula we derive that

$$
\begin{aligned}
\mathbb{E} \mid x_{\Delta}( & t)-\left.D\left(\bar{x}_{\Delta}(t-\tau), \bar{r}(t)\right)\right|^{p}-\left|\xi(0)-D\left(\xi(-\tau), r_{0}^{\Delta}\right)\right|^{p} \\
\leq & \mathbb{E} \int_{0}^{t} p\left|x_{\Delta}(s)-D\left(\bar{x}_{\Delta}(s-\tau), \bar{r}(s)\right)\right|^{p-2}\left[\left(x_{\Delta}(s)-D\left(\bar{x}_{\Delta}(s-\tau), \bar{r}(s)\right)\right)^{T}\right. \\
& \cdot f_{\Delta}\left(\mu(s), \bar{x}_{\Delta}(s), \bar{x}_{\Delta}(s-\tau), \bar{r}(s)\right) \\
& \left.+\frac{p-1}{2}\left|g_{\Delta}\left(\mu(s), \bar{x}_{\Delta}(s), \bar{x}_{\Delta}(s-\tau), \bar{r}(s)\right)\right|^{2}\right] d s \\
\leq & \mathbb{E} \int_{0}^{t} p\left|x_{\Delta}(s)-D\left(\bar{x}_{\Delta}(s-\tau), \bar{r}(s)\right)\right|^{p-2}\left(x_{\Delta}(s)-\bar{x}_{\Delta}(s)\right)^{T} \\
& \cdot f_{\Delta}\left(\mu(s), \bar{x}_{\Delta}(s), \bar{x}_{\Delta}(s-\tau), \bar{r}(s)\right) d s \\
& +\mathbb{E} \int_{0}^{t} p\left|x_{\Delta}(s)-D\left(\bar{x}_{\Delta}(s-\tau), \bar{r}(s)\right)\right|^{p-2}\left[\left(\bar{x}_{\Delta}(s)-D\left(\bar{x}_{\Delta}(s-\tau), \bar{r}(s)\right)\right)^{T}\right. \\
& \cdot f_{\Delta}\left(\mu(s), \bar{x}_{\Delta}(s), \bar{x}_{\Delta}(s-\tau), \bar{r}(s)\right) \\
& \left.+\frac{p-1}{2}\left|g_{\Delta}\left(\mu(s), \bar{x}_{\Delta}(s), \bar{x}_{\Delta}(s-\tau), \bar{r}(s)\right)\right|^{2}\right] d s \\
= & A_{1}+A_{2} .
\end{aligned}
$$

Let us first estimate $A_{1}$ :

$$
\begin{aligned}
A_{1} \leq & p \mathbb{E} \int_{0}^{t}\left|x_{\Delta}(s)-D\left(\bar{x}_{\Delta}(s-\tau), \bar{r}(s)\right)\right|^{p-2}\left(x_{\Delta}(s)-\bar{x}_{\Delta}(s)\right)^{T} \\
& \cdot \tilde{F}\left(\mu(s), \bar{x}_{\Delta}(s), \bar{x}_{\Delta}(s-\tau), \bar{r}(s)\right) d s \\
& +p \mathbb{E} \int_{0}^{t}\left|x_{\Delta}(s)-D\left(\bar{x}_{\Delta}(s-\tau), \bar{r}(s)\right)\right|^{p-2}\left(x_{\Delta}(s)-\bar{x}_{\Delta}(s)\right)^{T} \\
& \cdot F_{\Delta}\left(\mu(s), \bar{x}_{\Delta}(s), \bar{x}_{\Delta}(s-\tau), \bar{r}(s)\right) d s \\
=: & A_{11}+A_{12} .
\end{aligned}
$$

By Assumptions 2.3 and 3.1 and Young's inequality we derive that

$$
\begin{aligned}
A_{11} \leq & (p-2) \mathbb{E} \int_{0}^{t}\left|x_{\Delta}(s)-D\left(\bar{x}_{\Delta}(s-\tau), \bar{r}(s)\right)\right|^{p} d s \\
& +\frac{p}{2} \mathbb{E} \int_{0}^{t}\left|x_{\Delta}(s)-\bar{x}_{\Delta}(s)\right|^{\frac{p}{2}}\left|\tilde{F}\left(\mu(s), \bar{x}_{\Delta}(s), \bar{x}_{\Delta}(s-\tau), \bar{r}(s)\right)\right|^{\frac{p}{2}} d s \\
\leq & C \int_{0}^{t}\left(1+\mathbb{E}\left|x_{\Delta}(s)\right|^{p}+\mathbb{E}\left|\bar{x}_{\Delta}(s)\right|^{p}+\mathbb{E}\left|\bar{x}_{\Delta}(s-\tau)\right|^{p}\right) d s .
\end{aligned}
$$

Moreover, for any $t \in[0, T]$, there always is an integer $k \geq 0$ such that $t \in\left[t_{k}, t_{k+1}\right)$. By Hölder's inequality and BDG's inequality, we have

$$
\begin{aligned}
& \mathbb{E}\left|x_{\Delta}(t)-\bar{x}_{\Delta}(t)\right|^{\frac{p}{2}} \\
& \quad=\mathbb{E}\left|x_{\Delta}(t)-x_{\Delta}\left(t_{k}\right)\right|^{\frac{p}{2}}
\end{aligned}
$$




$$
\begin{aligned}
\leq & C \mathbb{E}\left|\int_{t_{k}}^{t} f_{\Delta}\left(\mu(s), \bar{x}_{\Delta}(s), \bar{x}_{\Delta}(s-\tau), \bar{r}(s)\right) d s\right|^{\frac{p}{2}} \\
& +C \mathbb{E}\left|\int_{t_{k}}^{t} g_{\Delta}\left(\mu(s), \bar{x}_{\Delta}(s), \bar{x}_{\Delta}(s-\tau), \bar{r}(s)\right) d B(s)\right|^{\frac{p}{2}} \\
\leq & C \Delta^{\frac{p}{2}-1} \mathbb{E} \int_{t_{k}}^{t}\left|\tilde{F}\left(\mu(s), \bar{x}_{\Delta}(s), \bar{x}_{\Delta}(s-\tau), \bar{r}(s)\right)\right|^{\frac{p}{2}} d s \\
& +C \Delta^{\frac{p}{2}-1} \mathbb{E} \int_{t_{k}}^{t}\left|F_{\Delta}\left(\mu(s), \bar{x}_{\Delta}(s), \bar{x}_{\Delta}(s-\tau), \bar{r}(s)\right)\right|^{\frac{p}{2}} d s \\
& +C \Delta^{\frac{p}{4}-1} \mathbb{E} \int_{t_{k}}^{t}\left|\tilde{G}\left(\mu(s), \bar{x}_{\Delta}(s), \bar{x}_{\Delta}(s-\tau), \bar{r}(s)\right)\right|^{\frac{p}{2}} d s \\
& +C \Delta^{\frac{p}{4}-1} \mathbb{E} \int_{t_{k}}^{t}\left|G G_{\Delta}\left(\mu(s), \bar{x}_{\Delta}(s), \bar{x}_{\Delta}(s-\tau), \bar{r}(s)\right)\right|^{\frac{p}{2}} d s \\
\leq & C \Delta^{\frac{p}{4}} h^{\frac{p}{2}}(\Delta)+C \Delta^{\frac{p}{4}}\left(1+\sup _{0 \leq s \leq t} \mathbb{E}\left|\bar{x}_{\Delta}(s)\right|^{\frac{p}{2}}+\sup _{0 \leq s \leq t} \mathbb{E}\left|\bar{x}_{\Delta}(s-\tau)\right|^{\frac{p}{2}}\right) .
\end{aligned}
$$

Thus, by (2.8), (2.10), and (3.19) and Young's inequality we get

$$
\begin{aligned}
A_{12} \leq & (p-2) \mathbb{E} \int_{0}^{t}\left|x_{\Delta}(s)-D\left(\bar{x}_{\Delta}(s-\tau), \bar{r}(s)\right)\right|^{p} d s \\
& +\frac{p}{2} \mathbb{E} \int_{0}^{t}\left|x_{\Delta}(s)-\bar{x}_{\Delta}(s)\right|^{\frac{p}{2}}\left|F_{\Delta}\left(\mu(s), \bar{x}_{\Delta}(s), \bar{x}_{\Delta}(s-\tau), \bar{r}(s)\right)\right|^{\frac{p}{2}} d s \\
\leq & (p-2) \mathbb{E} \int_{0}^{t}\left|x_{\Delta}(s)-D\left(\bar{x}_{\Delta}(s-\tau), \bar{r}(s)\right)\right|^{p} d s \\
& +\frac{p}{2} h^{\frac{p}{2}}(\Delta) \int_{0}^{t} \mathbb{E}\left|x_{\Delta}(s)-\bar{x}_{\Delta}(s)\right|^{\frac{p}{2}} d s \\
\leq & (p-2) \mathbb{E} \int_{0}^{t}\left|x_{\Delta}(s)-D\left(\bar{x}_{\Delta}(s-\tau), \bar{r}(s)\right)\right|^{p} d s \\
& +C h^{\frac{p}{2}}(\Delta) \Delta^{\frac{p}{4}} \int_{0}^{t}\left(1+h^{\frac{p}{2}}(\Delta)+\sup _{0 \leq l \leq s} \mathbb{E}\left|\bar{x}_{\Delta}(l)\right|^{\frac{p}{2}}+\sup _{0 \leq l \leq s} \mathbb{E}\left|\bar{x}_{\Delta}(l-\tau)\right|^{\frac{p}{2}}\right) d s \\
\leq & C \int_{0}^{t}\left(1+\sup _{0 \leq l \leq s} \mathbb{E}\left|x_{\Delta}(l)\right|^{p}+\sup _{0 \leq l \leq s} \mathbb{E}\left|\bar{x}_{\Delta}(l)\right|^{p}+\sup _{0 \leq l \leq s} \mathbb{E}\left|\bar{x}_{\Delta}(l-\tau)\right|^{p}\right) d s .
\end{aligned}
$$

Now, we are handling $A_{2}$. By Assumptions 2.3 and 3.6 and Hölder's inequality we get

$$
\begin{aligned}
A_{2} \leq & \mathbb{E} \int_{0}^{t} p\left|x_{\Delta}(s)-D\left(\bar{x}_{\Delta}(s-\tau), \bar{r}(s)\right)\right|^{p-2}\left[\left(\bar{K}_{8}+K_{8}\right)\left(1+\left|\bar{x}_{\Delta}(s)\right|^{2}\right)\right. \\
& \left.+\left(\bar{K}_{8}+\left|\bar{V}_{3}\left(\bar{x}_{\Delta}(s-\tau), 0\right)\right|\right)\left|\bar{x}_{\Delta}(s-\tau)\right|^{2}\right] d s \\
\leq & C \mathbb{E} \int_{0}^{t}\left|x_{\Delta}(s)-D\left(\bar{x}_{\Delta}(s-\tau), \bar{r}(s)\right)\right|^{p} d s+C \mathbb{E} \int_{0}^{t}\left(1+\left|\bar{x}_{\Delta}(s)\right|^{p}\right. \\
& \left.+\left|\bar{x}_{\Delta}(s-\tau)\right|^{p}\right) d s+C \mathbb{E} \int_{0}^{t}\left|\bar{V}_{3}\left(\bar{x}_{\Delta}(s-\tau), 0\right)\right|^{\frac{p}{2}}\left|\bar{x}_{\Delta}(s-\tau)\right|^{p} d s \\
\leq & C \int_{0}^{t}\left(1+\mathbb{E}\left|x_{\Delta}(s)\right|^{p}+\mathbb{E}\left|\bar{x}_{\Delta}(s)\right|^{p}+\mathbb{E}\left|\bar{x}_{\Delta}(s-\tau)\right|^{p}\right) d s
\end{aligned}
$$




$$
\begin{aligned}
& +C \int_{0}^{t}\left(\mathbb{E}\left|\bar{V}_{3}\left(\bar{x}_{\Delta}(s-\tau), 0\right)\right|^{p}+\mathbb{E}\left|\bar{x}_{\Delta}(s-\tau)\right|^{2 p}\right) d s \\
\leq & C \int_{0}^{t}\left(1+\mathbb{E}\left|x_{\Delta}(s)\right|^{p}+\mathbb{E}\left|\bar{x}_{\Delta}(s)\right|^{p}+\mathbb{E}\left|\bar{x}_{\Delta}(s-\tau)\right|^{p}\right) d s \\
& +C \int_{0}^{t} \mathbb{E}\left|\bar{x}_{\Delta}(s-\tau)\right|^{l_{* * p}} d s,
\end{aligned}
$$

where $l_{v *}=l_{v} \vee 2$. Inserting (3.17), (3.18), (3.20), and (3.21) into (3.16) yields that

$$
\begin{aligned}
& \mathbb{E}\left|x_{\Delta}(t)-D\left(\bar{x}_{\Delta}(t-\tau), \bar{r}(t)\right)\right|^{p} \\
& \quad \leq C\left(1+\int_{0}^{t} \sup _{0 \leq l \leq s} \mathbb{E}\left|x_{\Delta}(l)\right|^{p} d s+\int_{0}^{t} \sup _{0 \leq l \leq s} \mathbb{E}\left|x_{\Delta}(l-\tau)\right|^{l_{* * p} p} d s\right) .
\end{aligned}
$$

Therefore

$$
\begin{aligned}
& \sup _{0 \leq l \leq t} \mathbb{E}\left|x_{\Delta}(l)-D\left(\bar{x}_{\Delta}(l-\tau), \bar{r}(l)\right)\right|^{p} \\
& \quad \leq C\left(1+\int_{0}^{t} \sup _{0 \leq l \leq s} \mathbb{E}\left|x_{\Delta}(l)\right|^{p} d s+\int_{0}^{t} \sup _{0 \leq l \leq s} \mathbb{E}\left|x_{\Delta}(l-\tau)\right|^{l_{v * p}} d s\right) .
\end{aligned}
$$

Moreover, for any $c_{0}>0$,

$$
\begin{aligned}
\sup _{0 \leq l \leq t} \mathbb{E}\left|x_{\Delta}(l)\right|^{p}= & \sup _{0 \leq l \leq t} \mathbb{E}\left|x_{\Delta}(l)-D\left(\bar{x}_{\Delta}(l-\tau), \bar{r}(l)\right)+D\left(\bar{x}_{\Delta}(l-\tau), \bar{r}(l)\right)\right|^{p} \\
\leq & \left(1+c_{0}\right)^{p-1} \sup _{0 \leq l \leq t} \mathbb{E}\left|x_{\Delta}(l)-D\left(\bar{x}_{\Delta}(l-\tau), \bar{r}(l)\right)\right|^{p} \\
& +\left(\frac{1+c_{0}}{c_{0}}\right)^{p-1} K_{2}^{p}\left(\|\xi\|^{p}+\sup _{0 \leq l \leq t} \mathbb{E}\left|x_{\Delta}(l)\right|^{p}\right) .
\end{aligned}
$$

Then we can take $c_{0}$ large enough such that $\left(\frac{1+c_{0}}{c_{0}}\right)^{p-1} K_{2}^{p}<1$ for any $K_{2} \in(0,1)$. Thus

$$
\sup _{0 \leq l \leq t} \mathbb{E}\left|x_{\Delta}(l)\right|^{p} \leq c_{1} \sup _{0 \leq l \leq t} \mathbb{E}\left|x_{\Delta}(l)-D\left(\bar{x}_{\Delta}(l-\tau), \bar{r}(l)\right)\right|^{p}+c_{2}\|\xi\|^{p},
$$

where

$$
c_{1}=\frac{c_{0}^{p-1}\left(1+c_{0}\right)^{p-1}}{c_{0}^{p-1}-\left(1+c_{0}\right)^{p-1} K_{2}^{p}} \quad \text { and } \quad c_{2}=\frac{\left(1+c_{0}\right)^{p-1} K_{2}^{p}}{c_{0}^{p-1}-\left(1+c_{0}\right)^{p-1} K_{2}^{p}} .
$$

An application of Gronwall's inequality yields that

$$
\sup _{0 \leq l \leq t} \mathbb{E}\left|x_{\Delta}(l)\right|^{p} \leq C\left(1+\int_{0}^{t} \sup _{0 \leq l \leq s} \mathbb{E}\left|x_{\Delta}(l-\tau)\right|^{l_{v * p}} d s\right) .
$$

The following technique is similar to that in Theorem 2.1 of [35]. Define

$$
p_{i}=(\lfloor T / \tau\rfloor+2-i) p l_{\nu *}^{\lfloor T / \tau\rfloor+1-i}, \quad i=1,2, \ldots,\lfloor T / \tau\rfloor+1 .
$$

We can observe that

$$
p_{i+1} l_{v *}<p_{i} \quad \text { and } \quad p_{\lfloor T / \tau\rfloor+1}=p, \quad i=1,2, \ldots,\lfloor T / \tau\rfloor .
$$


By (3.26) and $\xi \in \mathscr{L}_{\mathcal{F}_{0}}^{p}\left([-\tau, 0] ; \mathbb{R}^{n}\right)$ we derive that

$$
\sup _{0 \leq l \leq \tau} \mathbb{E}\left|x_{\Delta}(l)\right|^{p_{1}} \leq C
$$

Then Hölder's inequality leads to

$$
\sup _{0 \leq l \leq 2 \tau} \mathbb{E}\left|x_{\Delta}(l)\right|^{p_{2}} \leq C\left(1+\int_{0}^{2 \tau} \sup _{0 \leq l \leq s}\left(\mathbb{E}\left|x_{\Delta}(l-\tau)\right|^{p_{1}}\right)^{\frac{l_{\nu * p_{2}}}{p_{1}}} d s\right) \leq C .
$$

The desired result follows by repeating this procedure. We complete the proof.

Lemma 3.10 Let Assumptions 2.3, 3.1, and 3.6 hold. Then for any $\Delta \in(0,1]$ and $t \in[0, T]$, we have

$$
\mathbb{E}\left|x_{\Delta}(t)-\bar{x}_{\Delta}(t)\right|^{p} \leq C \Delta^{\frac{p}{2}} h^{p}(\Delta) .
$$

Therefore

$$
\lim _{\Delta \rightarrow 0} \mathbb{E}\left|x_{\Delta}(t)-\bar{x}_{\Delta}(t)\right|^{p}=0
$$

Proof Fix any $\Delta \in(0,1]$. For any $t \in[0, T]$, there is an integer $k \geq 0$ such that $t \in\left[t_{k}, t_{k+1}\right)$. In the same way as in the proof of (3.19), we have

$$
\mathbb{E}\left|x_{\Delta}(t)-\bar{x}_{\Delta}(t)\right|^{p} \leq C \Delta^{\frac{p}{2}}\left(1+h^{p}(\Delta)+\mathbb{E}\left|\bar{x}_{\Delta}(t)\right|^{p}+\mathbb{E}\left|\bar{x}_{\Delta}(t-\tau)\right|^{p}\right)
$$

Then Lemma 3.9 gives that

$$
\mathbb{E}\left|x_{\Delta}(t)-\bar{x}_{\Delta}(t)\right|^{p} \leq C \Delta^{\frac{p}{2}} h^{p}(\Delta)
$$

We complete the proof.

Lemma 3.11 Let Assumptions 2.3, 3.1, and 3.6 hold. For any real number $L>\|\xi\|$, define the stopping time

$$
\tau_{\Delta, L}=\inf \left\{t \geq 0:\left|x_{\Delta}(t)\right| \geq L\right\} .
$$

Then we have

$$
\mathbb{P}\left(\tau_{\Delta, L} \leq T\right) \leq \frac{C}{L^{p}}
$$


Proof By Itô's formula and Assumption 3.6 we get

$$
\begin{aligned}
\mathbb{E} \mid x_{\Delta}( & \left.t \wedge \tau_{\Delta, L}\right)-\left.D\left(\bar{x}_{\Delta}\left(t \wedge \tau_{\Delta, L}-\tau\right), \bar{r}\left(t \wedge \tau_{\Delta, L}\right)\right)\right|^{p}-\left|\xi(0)-D\left(\xi(-\tau), r_{0}^{\Delta}\right)\right|^{p} \\
\leq & \mathbb{E} \int_{0}^{t \wedge \tau_{\Delta, L}} p\left|x_{\Delta}(s)-D\left(\bar{x}_{\Delta}(s-\tau), \bar{r}(s)\right)\right|^{p-2}\left[\left(x_{\Delta}(s)-D\left(\bar{x}_{\Delta}(s-\tau), \bar{r}(s)\right)\right)^{T}\right. \\
& \left.\cdot f_{\Delta}\left(\mu(s), \bar{x}_{\Delta}(s), \bar{x}_{\Delta}(s-\tau), \bar{r}(s)\right)+\frac{p-1}{2}\left|g_{\Delta}\left(\mu(s), \bar{x}_{\Delta}(s), \bar{x}_{\Delta}(s-\tau), \bar{r}(s)\right)\right|^{2}\right] d s \\
\leq & \mathbb{E} \int_{0}^{t \wedge \tau_{\Delta, L}} p\left|x_{\Delta}(s)-D\left(\bar{x}_{\Delta}(s-\tau), \bar{r}(s)\right)\right|^{p-2}\left[\left(\bar{x}_{\Delta}(s)-D\left(\bar{x}_{\Delta}(s-\tau), \bar{r}(s)\right)\right)^{T}\right. \\
& \left.\cdot f_{\Delta}\left(\mu(s), \bar{x}_{\Delta}(s), \bar{x}_{\Delta}(s-\tau), \bar{r}(s)\right)+\frac{p-1}{2}\left|g_{\Delta}\left(\mu(s), \bar{x}_{\Delta}(s), \bar{x}_{\Delta}(s-\tau), \bar{r}(s)\right)\right|^{2}\right] d s \\
& +\mathbb{E} \int_{0}^{t \wedge \tau_{\Delta, L}} p\left|x_{\Delta}(s)-D\left(\bar{x}_{\Delta}(s-\tau), \bar{r}(s)\right)\right|^{p-2}\left(x_{\Delta}(s)-\bar{x}_{\Delta}(s)\right)^{T} \\
& \cdot f_{\Delta}\left(\mu(s), \bar{x}_{\Delta}(s), \bar{x}_{\Delta}(s-\tau), \bar{r}(s)\right) d s \\
\leq & C \int_{0}^{t} \mathbb{E}\left|x_{\Delta}\left(s \wedge \tau_{\Delta, L}\right)-D\left(\bar{x}_{\Delta}\left(s \wedge \tau_{\Delta, L}-\tau\right), \bar{r}\left(s \wedge \tau_{\Delta, L}\right)\right)\right|^{p} d s \\
& +C \int_{0}^{t}\left(1+\mathbb{E}\left|\bar{x}_{\Delta}(s)\right|^{p}+\mathbb{E}\left|\bar{x}_{\Delta}(s-\tau)\right|^{p}\right) d s \\
& +C \mathbb{E} \int_{0}^{t \wedge \tau_{\Delta, L}}\left|\bar{V}_{3}\left(\bar{x}_{\Delta}(s-\tau), 0\right)\right|^{\frac{p}{2}}\left|\bar{x}_{\Delta}(s-\tau)\right|^{p} d s \\
& +C \mathbb{E} \int_{0}^{t}\left|x_{\Delta}(s)-\bar{x}_{\Delta}(s)\right|^{\frac{p}{2}}\left|f_{\Delta}\left(\mu(s), \bar{x}_{\Delta}(s), \bar{x}_{\Delta}(s-\tau), \bar{r}(s)\right)\right|^{\frac{p}{2}} d s . \\
&
\end{aligned}
$$

Note that

$$
\begin{aligned}
& \mathbb{E} \int_{0}^{t \wedge \tau_{\Delta, L}}\left|\bar{V}_{3}\left(\bar{x}_{\Delta}(s-\tau), 0\right)\right|^{\frac{p}{2}}\left|\bar{x}_{\Delta}(s-\tau)\right|^{p} d s \\
& \quad \leq \frac{1}{2} \int_{0}^{t} \mathbb{E}\left|\bar{V}_{3}\left(\bar{x}_{\Delta}\left(s \wedge \tau_{\Delta, L}-\tau\right), 0\right)\right|^{p} d s+\frac{1}{2} \int_{0}^{t} \mathbb{E}\left|\bar{x}_{\Delta}(s-\tau)\right|^{2 p} d s \\
& \quad \leq C \int_{0}^{t}\left(1+\mathbb{E}\left|\bar{x}_{\Delta}(s-\tau)\right|^{l_{\nu} p}+\mathbb{E}\left|\bar{x}_{\Delta}(s-\tau)\right|^{2 p}\right) d s
\end{aligned}
$$

and

$$
\begin{aligned}
& \mathbb{E} \int_{0}^{t}\left|x_{\Delta}(s)-\bar{x}_{\Delta}(s)\right|^{\frac{p}{2}}\left|f_{\Delta}\left(\mu(s), \bar{x}_{\Delta}(s), \bar{x}_{\Delta}(s-\tau), \bar{r}(s)\right)\right|^{\frac{p}{2}} d s \\
& \leq C \mathbb{E} \int_{0}^{t}\left|x_{\Delta}(s)-\bar{x}_{\Delta}(s)\right|^{\frac{p}{2}}\left|\tilde{F}\left(\mu(s), \bar{x}_{\Delta}(s), \bar{x}_{\Delta}(s-\tau), \bar{r}(s)\right)\right|^{\frac{p}{2}} d s \\
& \quad+C \mathbb{E} \int_{0}^{t}\left|x_{\Delta}(s)-\bar{x}_{\Delta}(s)\right|^{\frac{p}{2}}\left|F_{\Delta}\left(\mu(s), \bar{x}_{\Delta}(s), \bar{x}_{\Delta}(s-\tau), \bar{r}(s)\right)\right|^{\frac{p}{2}} d s \\
& \leq C \mathbb{E} \int_{0}^{t}\left(\left|x_{\Delta}(s)\right|^{\frac{p}{2}}+\left|\bar{x}_{\Delta}(s)\right|^{\frac{p}{2}}\right)\left(1+\left|\bar{x}_{\Delta}(s)\right|^{\frac{p}{2}}+\left|\bar{x}_{\Delta}(s-\tau)\right|^{\frac{p}{2}}\right) d s
\end{aligned}
$$




$$
\begin{aligned}
& +C h^{\frac{p}{2}}(\Delta) \int_{0}^{t} \mathbb{E}\left|x_{\Delta}(s)-\bar{x}_{\Delta}(s)\right|^{\frac{p}{2}} d s \\
\leq & C\left(1+\Delta^{\frac{p}{4}} h^{p}(\Delta)\right) \leq C,
\end{aligned}
$$

where (2.8), (2.10), (3.3), Young's inequality, and Lemma 3.9 were used. Then we obtain that

$$
\begin{aligned}
\mathbb{E}\left|x_{\Delta}\left(t \wedge \tau_{\Delta, L}\right)-D\left(\bar{x}_{\Delta}\left(t \wedge \tau_{\Delta, L}-\tau\right), \bar{r}\left(t \wedge \tau_{\Delta, L}\right)\right)\right|^{p} \\
\leq C\left(1+\int_{0}^{t} \mathbb{E}\left|\bar{x}_{\Delta}(s-\tau)\right|^{l_{\nu} * p} d s\right. \\
\left.\quad+\int_{0}^{t} \mathbb{E}\left|x_{\Delta}\left(s \wedge \tau_{\Delta, L}\right)-D\left(\bar{x}_{\Delta}\left(s \wedge \tau_{\Delta, L}-\tau\right), \bar{r}\left(s \wedge \tau_{\Delta, L}\right)\right)\right|^{p} d s\right),
\end{aligned}
$$

where $l_{v} *=l_{v} \vee 2$. Using the same technique as in Lemma 3.9 gives that

$$
\mathbb{E}\left|x_{\Delta}\left(T \wedge \tau_{\Delta, L}\right)-D\left(\bar{x}_{\Delta}\left(T \wedge \tau_{\Delta, L}-\tau\right), \bar{r}\left(T \wedge \tau_{\Delta, L}\right)\right)\right|^{p} \leq C
$$

We can get from (2.6) that

$$
\begin{aligned}
& \mathbb{I}_{\left\{\tau_{\Delta, L} \leq T\right\}}\left|x_{\Delta}\left(\tau_{\Delta, L}\right)-D\left(\bar{x}_{\Delta}\left(\tau_{\Delta, L}-\tau\right), \bar{r}\left(\tau_{\Delta, L}\right)\right)\right| \\
& \quad \geq \mathbb{I}_{\left\{\tau_{\Delta, L} \leq T\right\}}\left(\left|x_{\Delta}\left(\tau_{\Delta, L}\right)\right|-\left|D\left(\bar{x}_{\Delta}\left(\tau_{\Delta, L}-\tau\right), \bar{r}\left(\tau_{\Delta, L}\right)\right)\right|\right) \\
& \quad \geq L-K_{2} L .
\end{aligned}
$$

Hence we derive from (3.31) and (3.32) that

$$
\begin{aligned}
\mathbb{P}\left(\tau_{\Delta, L} \leq T\right) & \leq \frac{\mathbb{E}\left(\mathbb{I}_{\left\{\tau_{\Delta, L} \leq T\right\}}\left|x_{\Delta}\left(\tau_{\Delta, L}\right)-D\left(\bar{x}_{\Delta}\left(\tau_{\Delta, L}-\tau\right), \bar{r}\left(\tau_{\Delta, L}\right)\right)\right|^{p}\right)}{\left(1-K_{2}\right)^{p} L^{p}} \\
& \leq \frac{\mathbb{E}\left|x_{\Delta}\left(T \wedge \tau_{\Delta, L}\right)-D\left(\bar{x}_{\Delta}\left(T \wedge \tau_{\Delta, L}-\tau\right), \bar{r}\left(T \wedge \tau_{\Delta, L}\right)\right)\right|^{p}}{\left(1-K_{2}\right)^{p} L^{p}} \\
& \leq \frac{C}{\left(1-K_{2}\right)^{p} L^{p}} .
\end{aligned}
$$

Then the desired result follows. We complete the proof.

The following lemma can be proved in a similar way as Lemma 3.11 was, so we omit the proof.

Lemma 3.12 Let Assumptions 2.3, 3.1, and 3.3 hold. For any real number $L>\|\xi\|$, define the stopping time

$$
\tau_{L}=\inf \{t \geq 0:|x(t)| \geq L\}
$$

Then we have

$$
\mathbb{P}\left(\tau_{L} \leq T\right) \leq \frac{C}{L^{p}} .
$$


Lemma 3.13 Let Assumptions 2.2, 2.3, 3.1-3.4, and 3.6 hold. Assume that $q \in[2, \bar{q})$ and $p>\left(\beta+l_{v}+2\right) q$. Let $L>\|\xi\|$ be a real number, and let $\Delta \in(0,1]$ be sufficiently small such that $\varphi^{-1}(h(\Delta)) \geq L$. Then we have

$$
\mathbb{E}\left|x\left(T \wedge \rho_{\Delta, L}\right)-x_{\Delta}\left(T \wedge \rho_{\Delta, L}\right)\right|^{q} \leq C\left(\Delta^{\frac{q}{2}} h^{q}(\Delta) \vee \Delta^{q(\alpha \wedge \theta \wedge \sigma)}\right),
$$

where $\rho_{\Delta, L}:=\tau_{L} \wedge \tau_{\Delta, L}$ with $\tau_{L}, \tau_{\Delta, L}$ defined as before.

Proof For simplicity, we write $\rho_{\Delta, L}=\rho$. Denote $e_{\Delta}(t)=x(t)-D(x(t-\tau), r(t))-x_{\Delta}(t)+$ $D\left(\bar{x}_{\Delta}(t-\tau), \bar{r}(t)\right)$. For $0 \leq s \leq t \wedge \rho$, we can observe that

$$
|x(s)| \vee|x(s-\tau)| \vee\left|\bar{x}_{\Delta}(s)\right| \vee\left|\bar{x}_{\Delta}(s-\tau)\right| \leq L \leq \varphi^{-1}(h(\Delta)) .
$$

Recalling the definition of $F_{\Delta}$ and $G_{\Delta}$, we have

$$
\begin{aligned}
& F_{\Delta}\left(\mu(s), \bar{x}_{\Delta}(s), \bar{x}_{\Delta}(s-\tau), \bar{r}(s)\right)=F\left(\mu(s), \bar{x}_{\Delta}(s), \bar{x}_{\Delta}(s-\tau), \bar{r}(s)\right), \\
& G_{\Delta}\left(\mu(s), \bar{x}_{\Delta}(s), \bar{x}_{\Delta}(s-\tau), \bar{r}(s)\right)=G\left(\mu(s), \bar{x}_{\Delta}(s), \bar{x}_{\Delta}(s-\tau), \bar{r}(s)\right)
\end{aligned}
$$

for $0 \leq s \leq t \wedge \rho$. Hence we derive that

$$
\begin{aligned}
f_{\Delta} & \left(\mu(s), \bar{x}_{\Delta}(s), \bar{x}_{\Delta}(s-\tau), \bar{r}(s)\right) \\
& =\tilde{F}\left(\mu(s), \bar{x}_{\Delta}(s), \bar{x}_{\Delta}(s-\tau), \bar{r}(s)\right)+F_{\Delta}\left(\mu(s), \bar{x}_{\Delta}(s), \bar{x}_{\Delta}(s-\tau), \bar{r}(s)\right) \\
& =\tilde{F}\left(\mu(s), \bar{x}_{\Delta}(s), \bar{x}_{\Delta}(s-\tau), \bar{r}(s)\right)+F\left(\mu(s), \bar{x}_{\Delta}(s), \bar{x}_{\Delta}(s-\tau), \bar{r}(s)\right) \\
& =f\left(\mu(s), \bar{x}_{\Delta}(s), \bar{x}_{\Delta}(s-\tau), \bar{r}(s)\right) .
\end{aligned}
$$

Similarly,

$$
g_{\Delta}\left(\mu(s), \bar{x}_{\Delta}(s), \bar{x}_{\Delta}(s-\tau), \bar{r}(s)\right)=g\left(\mu(s), \bar{x}_{\Delta}(s), \bar{x}_{\Delta}(s-\tau), \bar{r}(s)\right) .
$$

By Itô’'s formula we get

$$
\begin{aligned}
& \mathbb{E}\left|e_{\Delta}(t \wedge \rho)\right|^{q} \\
& \leq \mathbb{E} \int_{0}^{t \wedge \rho} q\left|e_{\Delta}(s)\right|^{q-2}\left[e_{\Delta}^{T}(s)\left(f(s, x(s), x(s-\tau), r(s))-f_{\Delta}\left(\mu(s), \bar{x}_{\Delta}(s), \bar{x}_{\Delta}(s-\tau), \bar{r}(s)\right)\right)\right. \\
&\left.+\frac{q-1}{2}\left|g(s, x(s), x(s-\tau), r(s))-g_{\Delta}\left(\mu(s), \bar{x}_{\Delta}(s), \bar{x}_{\Delta}(s-\tau), \bar{r}(s)\right)\right|^{2}\right] d s \\
& \leq \mathbb{E} \int_{0}^{t \wedge \rho} q\left|e_{\Delta}(s)\right|^{q-2}\left[e_{\Delta}^{T}(s)\left(f(s, x(s), x(s-\tau), r(s))-f\left(\mu(s), \bar{x}_{\Delta}(s), \bar{x}_{\Delta}(s-\tau), \bar{r}(s)\right)\right)\right. \\
&\left.+\frac{q-1}{2}\left|g(s, x(s), x(s-\tau), r(s))-g\left(\mu(s), \bar{x}_{\Delta}(s), \bar{x}_{\Delta}(s-\tau), \bar{r}(s)\right)\right|^{2}\right] d s \\
& \leq \mathbb{E} \int_{0}^{t \wedge \rho} q\left|e_{\Delta}(s)\right|^{q-2}\left[\left(x(s)-D(x(s-\tau), r(s))-\bar{x}_{\Delta}(s)+D\left(\bar{x}_{\Delta}(s-\tau), r(s)\right)\right)^{T}\right. \\
& \quad \cdot\left(f(s, x(s), x(s-\tau), r(s))-f\left(\mu(s), \bar{x}_{\Delta}(s), \bar{x}_{\Delta}(s-\tau), \bar{r}(s)\right)\right)
\end{aligned}
$$




$$
\begin{aligned}
& \left.+\frac{q-1}{2}\left|g(s, x(s), x(s-\tau), r(s))-g\left(\mu(s), \bar{x}_{\Delta}(s), \bar{x}_{\Delta}(s-\tau), \bar{r}(s)\right)\right|^{2}\right] d s \\
& +\mathbb{E} \int_{0}^{t \wedge \rho} q\left|e_{\Delta}(s)\right|^{q-2}\left(\bar{x}_{\Delta}(s)-x_{\Delta}(s)+D\left(\bar{x}_{\Delta}(s-\tau), \bar{r}(s)\right)-D\left(\bar{x}_{\Delta}(s-\tau), r(s)\right)\right)^{T} \\
& \cdot\left(f(s, x(s), x(s-\tau), r(s))-f\left(\mu(s), \bar{x}_{\Delta}(s), \bar{x}_{\Delta}(s-\tau), \bar{r}(s)\right)\right) d s \\
& \leq \mathbb{E} \int_{0}^{t \wedge \rho} q\left|e_{\Delta}(s)\right|^{q-2}\left[\left(x(s)-D(x(s-\tau), r(s))-\bar{x}_{\Delta}(s)+D\left(\bar{x}_{\Delta}(s-\tau), r(s)\right)\right)^{T}\right. \\
& \cdot\left(f(s, x(s), x(s-\tau), r(s))-f\left(s, \bar{x}_{\Delta}(s), \bar{x}_{\Delta}(s-\tau), r(s)\right)\right) \\
& \left.+\frac{q-1}{2}\left|g(s, x(s), x(s-\tau), r(s))-g\left(\mu(s), \bar{x}_{\Delta}(s), \bar{x}_{\Delta}(s-\tau), \bar{r}(s)\right)\right|^{2}\right] d s \\
& +\mathbb{E} \int_{0}^{t \wedge \rho} q\left|e_{\Delta}(s)\right|^{q-2}\left(x(s)-\bar{x}_{\Delta}(s)+D\left(\bar{x}_{\Delta}(s-\tau), r(s)\right)-D(x(s-\tau), r(s))\right)^{T} \\
& \cdot\left(f\left(s, \bar{x}_{\Delta}(s), \bar{x}_{\Delta}(s-\tau), r(s)\right)-f\left(\mu(s), \bar{x}_{\Delta}(s), \bar{x}_{\Delta}(s-\tau), \bar{r}(s)\right)\right) d s \\
& +\mathbb{E} \int_{0}^{t \wedge \rho} q\left|e_{\Delta}(s)\right|^{q-2}\left(\bar{x}_{\Delta}(s)-x_{\Delta}(s)+D\left(\bar{x}_{\Delta}(s-\tau), \bar{r}(s)\right)-D\left(\bar{x}_{\Delta}(s-\tau), r(s)\right)\right)^{T} \\
& \cdot\left(f(s, x(s), x(s-\tau), r(s))-f\left(s, \bar{x}_{\Delta}(s), \bar{x}_{\Delta}(s-\tau), r(s)\right)\right) d s \\
& +\mathbb{E} \int_{0}^{t \wedge \rho} q\left|e_{\Delta}(s)\right|^{q-2}\left(\bar{x}_{\Delta}(s)-x_{\Delta}(s)+D\left(\bar{x}_{\Delta}(s-\tau), \bar{r}(s)\right)-D\left(\bar{x}_{\Delta}(s-\tau), r(s)\right)\right)^{T} \\
& +\left(f\left(s, \bar{x}_{\Delta}(s), \bar{x}_{\Delta}(s-\tau), r(s)\right)-f\left(\mu(s), \bar{x}_{\Delta}(s), \bar{x}_{\Delta}(s-\tau), \bar{r}(s)\right)\right) d s .
\end{aligned}
$$

Note that

$$
\begin{aligned}
& \frac{q-1}{2}\left|g(s, x(s), x(s-\tau), r(s))-g\left(\mu(s), \bar{x}_{\Delta}(s), \bar{x}_{\Delta}(s-\tau), \bar{r}(s)\right)\right|^{2} \\
& \quad \leq \frac{\bar{q}-1}{2}\left|g(s, x(s), x(s-\tau), r(s))-g\left(s, \bar{x}_{\Delta}(s), \bar{x}_{\Delta}(s-\tau), r(s)\right)\right|^{2} \\
& \quad+\frac{(q-1)(\bar{q}-1)}{2(\bar{q}-q)}\left|g\left(s, \bar{x}_{\Delta}(s), \bar{x}_{\Delta}(s-\tau), r(s)\right)-g\left(\mu(s), \bar{x}_{\Delta}(s), \bar{x}_{\Delta}(s-\tau), \bar{r}(s)\right)\right|^{2} .
\end{aligned}
$$

Hence

$$
\begin{aligned}
\mathbb{E}\left|e_{\Delta}(t \wedge \rho)\right|^{q} \\
\leq \mathbb{E} \int_{0}^{t \wedge \rho} q\left|e_{\Delta}(s)\right|^{q-2}\left[\left(x(s)-D(x(s-\tau), r(s))-\bar{x}_{\Delta}(s)+D\left(\bar{x}_{\Delta}(s-\tau), r(s)\right)\right)^{T}\right. \\
\quad \cdot\left(f(s, x(s), x(s-\tau), r(s))-f\left(s, \bar{x}_{\Delta}(s), \bar{x}_{\Delta}(s-\tau), r(s)\right)\right) \\
\left.\quad+\frac{q-1}{2}\left|g(s, x(s), x(s-\tau), r(s))-g\left(s, \bar{x}_{\Delta}(s), \bar{x}_{\Delta}(s-\tau), r(s)\right)\right|^{2}\right] d s \\
\quad+\mathbb{E} \int_{0}^{t \wedge \rho} q\left|e_{\Delta}(s)\right|^{q-2}\left(x(s)-\bar{x}_{\Delta}(s)+D\left(\bar{x}_{\Delta}(s-\tau), r(s)\right)-D(x(s-\tau), r(s))\right)^{T} \\
\quad \cdot\left(f\left(s, \bar{x}_{\Delta}(s), \bar{x}_{\Delta}(s-\tau), r(s)\right)-f\left(\mu(s), \bar{x}_{\Delta}(s), \bar{x}_{\Delta}(s-\tau), \bar{r}(s)\right)\right) d s \\
\quad+\mathbb{E} \int_{0}^{t \wedge \rho} q\left|e_{\Delta}(s)\right|^{q-2}\left(\bar{x}_{\Delta}(s)-x_{\Delta}(s)+D\left(\bar{x}_{\Delta}(s-\tau), \bar{r}(s)\right)\right.
\end{aligned}
$$




$$
\begin{aligned}
& \left.-D\left(\bar{x}_{\Delta}(s-\tau), r(s)\right)\right)^{T} \\
& \cdot\left(f(s, x(s), x(s-\tau), r(s))-f\left(s, \bar{x}_{\Delta}(s), \bar{x}_{\Delta}(s-\tau), r(s)\right)\right) d s \\
+ & \mathbb{E} \int_{0}^{t \wedge \rho} q\left|e_{\Delta}(s)\right|^{q-2}\left(\bar{x}_{\Delta}(s)-x_{\Delta}(s)+D\left(\bar{x}_{\Delta}(s-\tau), \bar{r}(s)\right)-D\left(\bar{x}_{\Delta}(s-\tau), r(s)\right)\right)^{T} \\
& \cdot\left(f\left(s, \bar{x}_{\Delta}(s), \bar{x}_{\Delta}(s-\tau), r(s)\right)-f\left(\mu(s), \bar{x}_{\Delta}(s), \bar{x}_{\Delta}(s-\tau), \bar{r}(s)\right)\right) d s \\
& +\mathbb{E} \int_{0}^{t \wedge \rho} \frac{(q-1)(\bar{q}-1)}{2(\bar{q}-q)} q\left|e_{\Delta}(s)\right|^{q-2} \\
& \cdot\left|g\left(s, \bar{x}_{\Delta}(s), \bar{x}_{\Delta}(s-\tau), r(s)\right)-g\left(\mu(s), \bar{x}_{\Delta}(s), \bar{x}_{\Delta}(s-\tau), \bar{r}(s)\right)\right|^{2} d s \\
=: & B_{1}+B_{2}+B_{3}+B_{4}+B_{5} .
\end{aligned}
$$

By Hölder's inequality, Assumptions 2.2 and 3.2, and Lemmas 3.9 and 3.10 we get

$$
\begin{aligned}
& B_{1} \leq C \mathbb{E} \int_{0}^{t \wedge \rho}\left|e_{\Delta}(s)\right|^{q} d s+C \mathbb{E} \int_{0}^{t \wedge \rho}\left|x(s)-\bar{x}_{\Delta}(s)\right|^{q} d s \\
& +C \mathbb{E} \int_{0}^{t \wedge \rho}\left(\bar{K}_{4}+\left|\bar{V}_{1}\left(x(s-\tau), \bar{x}_{\Delta}(s-\tau)\right)\right|\right)^{\frac{q}{2}}\left|x(s-\tau)-\bar{x}_{\Delta}(s-\tau)\right|^{q} d s \\
& \leq C\left(\mathbb{E} \int_{0}^{t \wedge \rho}\left|e_{\Delta}(s)\right|^{q} d s+\int_{0}^{t} \mathbb{E}\left|x(s \wedge \rho)-x_{\Delta}(s \wedge \rho)\right|^{q} d s\right. \\
& +\int_{0}^{T} \mathbb{E}\left|x_{\Delta}(s)-\bar{x}_{\Delta}(s)\right|^{q} d s+\int_{-\tau}^{0}|\xi(s)-\xi(\lfloor s / \Delta\rfloor \Delta)|^{q} d s \\
& +\int_{0}^{T}\left(\mathbb{E}\left|\bar{V}_{1}\left(x(s-\tau), \bar{x}_{\Delta}(s-\tau)\right)\right|^{q}\right)^{\frac{1}{2}}\left(\mathbb{E}\left|x_{\Delta}(s-\tau)-\bar{x}_{\Delta}(s-\tau)\right|^{2 q}\right)^{\frac{1}{2}} d s \\
& \left.+\mathbb{E} \int_{0}^{t \wedge \rho}\left|\bar{V}_{1}\left(x(s-\tau), \bar{x}_{\Delta}(s-\tau)\right)\right|^{\frac{q}{2}}\left|x(s-\tau)-x_{\Delta}(s-\tau)\right|^{q} d s\right) \\
& \leq C\left(\mathbb{E} \int_{0}^{t \wedge \rho}\left|e_{\Delta}(s)\right|^{q} d s+\int_{0}^{t} \mathbb{E}\left|x(s \wedge \rho)-x_{\Delta}(s \wedge \rho)\right|^{q} d s+\Delta^{\frac{q}{2}} h^{q}(\Delta)+\Delta^{q \alpha}\right. \\
& +\int_{0}^{t}\left(\mathbb{E}\left|\bar{V}_{1}\left(x(s \wedge \rho-\tau), \bar{x}_{\Delta}(s \wedge \rho-\tau)\right)\right|^{q}\right)^{\frac{1}{2}} \\
& \left.\times\left(\mathbb{E}\left|x(s \wedge \rho-\tau)-x_{\Delta}(s \wedge \rho-\tau)\right|^{2 q}\right)^{\frac{1}{2}} d s\right) \\
& \leq C\left(\mathbb{E} \int_{0}^{t \wedge \rho}\left|e_{\Delta}(s)\right|^{q} d s+\int_{0}^{t} \mathbb{E}\left|x(s \wedge \rho)-x_{\Delta}(s \wedge \rho)\right|^{q} d s+\Delta^{\frac{q}{2}} h^{q}(\Delta)+\Delta^{q \alpha}\right. \\
& \left.+\int_{0}^{t}\left(\mathbb{E}\left|x(s \wedge \rho-\tau)-x_{\Delta}(s \wedge \rho-\tau)\right|^{2 q}\right)^{\frac{1}{2}} d s\right) \text {. }
\end{aligned}
$$

As for $B_{2}$, we derive from Assumptions 2.3 and 3.4 that

$$
\begin{aligned}
B_{2} \leq & C \mathbb{E} \int_{0}^{t \wedge \rho}\left|x(s)-\bar{x}_{\Delta}(s)+D\left(\bar{x}_{\Delta}(s-\tau), r(s)\right)-D(x(s-\tau), r(s))\right|^{q} d s \\
& +C \mathbb{E} \int_{0}^{t \wedge \rho}\left|f\left(s, \bar{x}_{\Delta}(s), \bar{x}_{\Delta}(s-\tau), r(s)\right)-f\left(\mu(s), \bar{x}_{\Delta}(s), \bar{x}_{\Delta}(s-\tau), \bar{r}(s)\right)\right|^{q} d s \\
& +C \mathbb{E} \int_{0}^{t \wedge \rho}\left|e_{\Delta}(s)\right|^{q} d s
\end{aligned}
$$




$$
\begin{aligned}
\leq & C \mathbb{E} \int_{0}^{t \wedge \rho}\left(\left|x(s)-\bar{x}_{\Delta}(s)\right|^{q}+\left|D\left(\bar{x}_{\Delta}(s-\tau), r(s)\right)-D(x(s-\tau), r(s))\right|^{q}\right) d s \\
& +C \mathbb{E} \int_{0}^{t \wedge \rho}\left(\left|f\left(s, \bar{x}_{\Delta}(s), \bar{x}_{\Delta}(s-\tau), r(s)\right)-f\left(\mu(s), \bar{x}_{\Delta}(s), \bar{x}_{\Delta}(s-\tau), r(s)\right)\right|^{q}\right. \\
& \left.+\left|f\left(\mu(s), \bar{x}_{\Delta}(s), \bar{x}_{\Delta}(s-\tau), r(s)\right)-f\left(\mu(s), \bar{x}_{\Delta}(s), \bar{x}_{\Delta}(s-\tau), \bar{r}(s)\right)\right|^{q}\right) d s \\
& +C \mathbb{E} \int_{0}^{t \wedge \rho}\left|e_{\Delta}(s)\right|^{q} d s \\
\leq & C \mathbb{E} \int_{0}^{t \wedge \rho}\left|e_{\Delta}(s)\right|^{q} d s+C \mathbb{E} \int_{0}^{t \wedge \rho}\left(\left|x(s)-\bar{x}_{\Delta}(s)\right|^{q}+\left|x(s-\tau)-\bar{x}_{\Delta}(s-\tau)\right|^{q}\right) d s \\
& +C \mathbb{E} \int_{0}^{t \wedge \rho}\left(1+\left|\bar{x}_{\Delta}(s)\right|^{q \beta+q}+\left|\bar{x}_{\Delta}(s-\tau)\right|^{q \beta+q}\right) \Delta^{q \theta} d s \\
& \left.+C \mathbb{E} \int_{0}^{t \wedge \rho}\left|f\left(\mu(s), \bar{x}_{\Delta}(s), \bar{x}_{\Delta}(s-\tau), r(s)\right)-f\left(\mu(s), \bar{x}_{\Delta}(s), \bar{x}_{\Delta}(s-\tau), \bar{r}(s)\right)\right|^{q}\right) d s .
\end{aligned}
$$

From (3.38) we get

$$
\begin{aligned}
& \mathbb{E} \int_{0}^{t \wedge \rho}\left(\left|x(s)-\bar{x}_{\Delta}(s)\right|^{q}+\left|x(s-\tau)-\bar{x}_{\Delta}(s-\tau)\right|^{q}\right) d s \\
& \quad \leq C \int_{0}^{t} \mathbb{E}\left|x(s \wedge \rho)-x_{\Delta}(s \wedge \rho)\right|^{q} d s+C\left(\Delta^{\frac{q}{2}} h^{q}(\Delta)+\Delta^{q \alpha}\right),
\end{aligned}
$$

and we have

$$
\mathbb{E} \int_{0}^{t \wedge \rho}\left(1+\left|\bar{x}_{\Delta}(s)\right|^{q \beta+q}+\left|\bar{x}_{\Delta}(s-\tau)\right|^{q \beta+q}\right) \Delta^{q \theta} d s \leq C \Delta^{q \theta} .
$$

Moreover, let $j$ be the integer part of $T / \Delta$. Then

$$
\begin{aligned}
\mathbb{E} \int_{0}^{t \wedge \rho}\left|f\left(\mu(s), \bar{x}_{\Delta}(s), \bar{x}_{\Delta}(s-\tau), r(s)\right)-f\left(\mu(s), \bar{x}_{\Delta}(s), \bar{x}_{\Delta}(s-\tau), \bar{r}(s)\right)\right|^{q} d s \\
=\sum_{k=0}^{j} \mathbb{E} \int_{t_{k}}^{t_{k+1}} \mid f\left(\mu(s), \bar{x}_{\Delta}(s), \bar{x}_{\Delta}(s-\tau), r(s)\right) \\
\quad-\left.f\left(\mu(s), \bar{x}_{\Delta}(s), \bar{x}_{\Delta}(s-\tau), r\left(t_{k}\right)\right)\right|^{q} \mathbb{I}_{[0, t \wedge \rho]}(s) d s \\
\leq 2^{q-1} \sum_{k=0}^{j} \mathbb{E} \int_{t_{k}}^{t_{k+1}}\left(\left|f\left(\mu(s), \bar{x}_{\Delta}(s), \bar{x}_{\Delta}(s-\tau), r(s)\right)\right|^{q}\right. \\
\left.\quad+\left|f\left(\mu(s), \bar{x}_{\Delta}(s), \bar{x}_{\Delta}(s-\tau), r\left(t_{k}\right)\right)\right|^{q}\right) \mathbb{I}_{[0, t \wedge \rho]}(s) \mathbb{I}_{\left\{r(s) \neq r\left(t_{k}\right)\right\}} d s \\
\leq C \sum_{k=0}^{j} \int_{t_{k}}^{t_{k+1}} \mathbb{E}\left(\mathbb{E}\left[\left(1+\left|\bar{x}_{\Delta}(s)\right|^{q}+\left|\bar{x}_{\Delta}(s-\tau)\right|^{q}+h^{q}(\Delta)\right) \mathbb{I}_{\left\{r(s) \neq r\left(t_{k}\right)\right\}} \mid r\left(t_{k}\right)\right]\right) d s,
\end{aligned}
$$

where in the last step, we used the fact that $\bar{x}_{\Delta}(s)$ and $\bar{x}_{\Delta}(s-\tau)$ are conditionally independent of $\mathbb{I}_{\left\{r(s) \neq r\left(t_{k}\right)\right\}}$ with respect to the $\sigma$-algebra generated by $r\left(t_{k}\right)$. Applying the Markov 
property yields that

$$
\begin{aligned}
\mathbb{E} & \left.\mathbb{I}_{\left\{r(s) \neq r\left(t_{k}\right)\right\}} \mid r\left(t_{k}\right)\right) \\
& =\sum_{i \in \mathbb{S}} \mathbb{I}_{\left\{r\left(t_{k}\right)=i\right\}} \mathbb{P}\left(r(s) \neq i \mid r\left(t_{k}\right)=i\right) \\
& =\sum_{i \in \mathbb{S}} \mathbb{I}_{\left\{r\left(t_{k}\right)=i\right\}} \sum_{j \neq i}\left(\gamma_{i j}\left(s-t_{k}\right)+o\left(s-t_{k}\right)\right) \\
& \leq \max _{0 \leq i \leq N}\left(-\gamma_{i i} \Delta+o(\Delta)\right) \sum_{i \in \mathbb{S}} \mathbb{I}_{\left\{r\left(t_{k}\right)=i\right\}} \\
& \leq C \Delta+o(\Delta) .
\end{aligned}
$$

By Lemma 3.9 we have

$$
\begin{aligned}
& \mathbb{E} \int_{0}^{t \wedge \rho}\left|f\left(\mu(s), \bar{x}_{\Delta}(s), \bar{x}_{\Delta}(s-\tau), r(s)\right)-f\left(\mu(s), \bar{x}_{\Delta}(s), \bar{x}_{\Delta}(s-\tau), \bar{r}(s)\right)\right|^{q} d s \\
& \quad \leq(C \Delta+o(\Delta)) \sum_{k=0}^{j} \int_{t_{k}}^{t_{k+1}}\left(1+\mathbb{E}\left|\bar{x}_{\Delta}(s)\right|^{q}+\mathbb{E}\left|\bar{x}_{\Delta}(s-\tau)\right|^{q}+h^{q}(\Delta)\right) d s \\
& \quad \leq h^{q}(\Delta)(C \Delta+o(\Delta)) .
\end{aligned}
$$

Inserting (3.40), (3.41), and (3.44) into (3.39) gives that

$$
\begin{aligned}
B_{2} \leq & C\left(\mathbb{E} \int_{0}^{t \wedge \rho}\left|e_{\Delta}(s)\right|^{q} d s+\int_{0}^{t} \mathbb{E}\left|x(s \wedge \rho)-x_{\Delta}(s \wedge \rho)\right|^{q} d s\right. \\
& \left.+\Delta^{\frac{q}{2}} h^{q}(\Delta)+\Delta^{q \alpha}+\Delta^{q \theta}+o(\Delta)\right) .
\end{aligned}
$$

In addition, we obtain from Assumptions 2.2 and 3.1 and Lemmas 3.5, 3.9, and 3.10 that

$$
\begin{aligned}
B_{3} \leq & C \mathbb{E} \int_{0}^{t \wedge \rho}\left|e_{\Delta}(s)\right|^{q} d s \\
& +C \mathbb{E} \int_{0}^{t \wedge \rho}\left|\bar{x}_{\Delta}(s)-x_{\Delta}(s)+D\left(\bar{x}_{\Delta}(s-\tau), \bar{r}(s)\right)-D\left(\bar{x}_{\Delta}(s-\tau), r(s)\right)\right|^{q} d s \\
& +C \mathbb{E} \int_{0}^{t \wedge \rho}\left|f(s, x(s), x(s-\tau), r(s))-f\left(s, \bar{x}_{\Delta}(s), \bar{x}_{\Delta}(s-\tau), r(s)\right)\right|^{q} d s \\
\leq & C \mathbb{E} \int_{0}^{t \wedge \rho}\left|e_{\Delta}(s)\right|^{q} d s+C \int_{0}^{T} \mathbb{E}\left|D\left(\bar{x}_{\Delta}(s-\tau), r(s)\right)-D\left(\bar{x}_{\Delta}(s-\tau), \bar{r}(s)\right)\right|^{q} d s \\
& +C \mathbb{E} \int_{0}^{t \wedge \rho}\left(1+|x(s)|^{q \beta}+|x(s-\tau)|^{q \beta}+\left|\bar{x}_{\Delta}(s)\right|^{q \beta}+\left|\bar{x}_{\Delta}(s-\tau)\right|^{q \beta}\right) \\
& +\left(\left|x(s)-\bar{x}_{\Delta}(s)\right|^{q}+\left|x(s-\tau)-\bar{x}_{\Delta}(s-\tau)\right|^{q}\right) d s+C \int_{0}^{T} \mathbb{E}\left|\bar{x}_{\Delta}(s)-x_{\Delta}(s)\right|^{q} d s \\
\leq & C\left(\mathbb{E} \int_{0}^{t \wedge \rho}\left|e_{\Delta}(s)\right|^{q} d s+\int_{0}^{T} \mathbb{E}\left|D\left(\bar{x}_{\Delta}(s-\tau), r(s)\right)-D\left(\bar{x}_{\Delta}(s-\tau), \bar{r}(s)\right)\right|^{q} d s\right. \\
& \left.+\int_{0}^{t} \mathbb{E}\left|x(s \wedge \rho)-x_{\Delta}(s \wedge \rho)\right|^{q} d s+\Delta^{\frac{q}{2}} h^{q}(\Delta)+\Delta^{q \alpha}\right) .
\end{aligned}
$$


Furthermore, let $j$ be the integer part of $T / \Delta$. Then by Assumption 2.3 and Lemma 3.9 we have

$$
\begin{aligned}
\sup _{0 \leq s \leq T} \mathbb{E}\left|D\left(\bar{x}_{\Delta}(s-\tau), r(s)\right)-D\left(\bar{x}_{\Delta}(s-\tau), \bar{r}(s)\right)\right|^{q} \\
\leq \max _{0 \leq k \leq j}\left(\sup _{t_{k} \leq s \leq t_{k+1}} \mathbb{E}\left|D\left(\bar{x}_{\Delta}(s-\tau), r(s)\right)-D\left(\bar{x}_{\Delta}(s-\tau), \bar{r}(s)\right)\right|^{q}\right) \\
\leq 2 \max _{0 \leq k \leq j}\left(\sup _{t_{k} \leq s \leq t_{k+1}} \mathbb{E}\left[\left|D\left(\bar{x}_{\Delta}(s-\tau), r(s)\right)-D\left(\bar{x}_{\Delta}(s-\tau), \bar{r}(s)\right)\right|^{q} \mathbb{I}_{\left\{r(s) \neq r\left(t_{k}\right)\right\}}\right]\right) \\
\leq C \max _{0 \leq k \leq j}\left(\operatorname { s u p } _ { t _ { k } \leq s \leq t _ { k + 1 } } \mathbb { E } \left[\left(\left|D\left(\bar{x}_{\Delta}(s-\tau), r(s)\right)\right|^{q}\right.\right.\right. \\
\left.\left.\left.\quad+\left|D\left(\bar{x}_{\Delta}(s-\tau), \bar{r}(s)\right)\right|^{q}\right) \mathbb{I}_{\left\{r(s) \neq r\left(t_{k}\right)\right\}}\right]\right) \\
\leq C \max _{0 \leq k \leq j}\left(1+\sup _{t_{k} \leq s \leq t_{k+1}} \mathbb{E}\left|\bar{x}_{\Delta}(s-\tau)\right|^{q}\right) \mathbb{E}\left(\mathbb{I}_{\left\{r(s) \neq r\left(t_{k}\right)\right\}}\right) \\
\leq C \mathbb{E}\left(\mathbb{I}_{\left\{r(s) \neq r\left(t_{k}\right)\right\}} .\right.
\end{aligned}
$$

By (3.43) we get

$$
\mathbb{E}\left(\mathbb{I}_{\left\{r(s) \neq r\left(t_{k}\right)\right\}}\right)=\mathbb{E}\left[\mathbb{E}\left(\mathbb{I}_{\left\{r(s) \neq r\left(t_{k}\right)\right\}} \mid r\left(t_{k}\right)\right)\right] \leq C \Delta+o(\Delta) .
$$

Hence, for any $s \in[0, T]$, we derive that

$$
\begin{aligned}
& \mathbb{E}\left|D\left(\bar{x}_{\Delta}(s-\tau), r(s)\right)-D\left(\bar{x}_{\Delta}(s-\tau), \bar{r}(s)\right)\right|^{q} \\
& \quad \leq \sup _{0 \leq s \leq T} \mathbb{E}\left|D\left(\bar{x}_{\Delta}(s-\tau), r(s)\right)-D\left(\bar{x}_{\Delta}(s-\tau), \bar{r}(s)\right)\right|^{q} \\
& \quad \leq C \Delta+o(\Delta) .
\end{aligned}
$$

Inserting (3.47) into (3.46) gives that

$$
\begin{aligned}
B_{3} \leq & C\left(\mathbb{E} \int_{0}^{t \wedge \rho}\left|e_{\Delta}(s)\right|^{q} d s+\int_{0}^{t} \mathbb{E}\left|x(s \wedge \rho)-x_{\Delta}(s \wedge \rho)\right|^{q} d s\right. \\
& \left.+\Delta^{\frac{q}{2}} h^{q}(\Delta)+\Delta^{q \alpha}+o(\Delta)\right) .
\end{aligned}
$$

Similarly to $B_{2}$ and $B_{3}$, we easily derive that

$$
\begin{aligned}
B_{4} \leq & C\left(\mathbb{E} \int_{0}^{t \wedge \rho}\left|e_{\Delta}(s)\right|^{q} d s+\mathbb{E} \int_{0}^{t \wedge \rho}\left|\bar{x}_{\Delta}(s)-x_{\Delta}(s)\right|^{q} d s\right. \\
& +\mathbb{E} \int_{0}^{t \wedge \rho}\left|D\left(\bar{x}_{\Delta}(s-\tau), \bar{r}(s)\right)-D\left(\bar{x}_{\Delta}(s-\tau), r(s)\right)\right|^{q} d s \\
& +\mathbb{E} \int_{0}^{t \wedge \rho}\left|f\left(s, \bar{x}_{\Delta}(s), \bar{x}_{\Delta}(s-\tau), r(s)\right)-f\left(\mu(s), \bar{x}_{\Delta}(s), \bar{x}_{\Delta}(s-\tau), r(s)\right)\right|^{q} d s \\
& \left.+\mathbb{E} \int_{0}^{t \wedge \rho}\left|f\left(\mu(s), \bar{x}_{\Delta}(s), \bar{x}_{\Delta}(s-\tau), r(s)\right)-f\left(\mu(s), \bar{x}_{\Delta}(s), \bar{x}_{\Delta}(s-\tau), \bar{r}(s)\right)\right|^{q} d s\right) \\
\leq & C\left(\mathbb{E} \int_{0}^{t \wedge \rho}\left|e_{\Delta}(s)\right|^{q} d s+\Delta^{\frac{q}{2}} h^{q}(\Delta)+\Delta^{q \theta}+o(\Delta)\right)
\end{aligned}
$$


and

$$
\begin{aligned}
B_{5} \leq & C\left(\mathbb{E} \int_{0}^{t \wedge \rho}\left|e_{\Delta}(s)\right|^{q} d s\right. \\
& +\mathbb{E} \int_{0}^{t \wedge \rho}\left|g\left(s, \bar{x}_{\Delta}(s), \bar{x}_{\Delta}(s-\tau), r(s)\right)-g\left(\mu(s), \bar{x}_{\Delta}(s), \bar{x}_{\Delta}(s-\tau), r(s)\right)\right|^{q} d s \\
& \left.+\mathbb{E} \int_{0}^{t \wedge \rho}\left|g\left(\mu(s), \bar{x}_{\Delta}(s), \bar{x}_{\Delta}(s-\tau), r(s)\right)-g\left(\mu(s), \bar{x}_{\Delta}(s), \bar{x}_{\Delta}(s-\tau), \bar{r}(s)\right)\right|^{q} d s\right) \\
\leq & C\left(\mathbb{E} \int_{0}^{t \wedge \rho}\left|e_{\Delta}(s)\right|^{q} d s+\Delta^{\frac{q}{2}} h^{q}(\Delta)+\Delta^{q \sigma}+o(\Delta)\right) .
\end{aligned}
$$

Substituting (3.38), (3.45), (3.48), (3.49), and (3.50) into (3.37) yields that

$$
\begin{aligned}
\mathbb{E} \mid e_{\Delta}( & t \wedge \rho)\left.\right|^{q} \\
\leq & C\left(\int_{0}^{t} \mathbb{E}\left|e_{\Delta}(s \wedge \rho)\right|^{q} d s+\int_{0}^{t} \sup _{0 \leq l \leq s} \mathbb{E}\left|x(l \wedge \rho)-x_{\Delta}(l \wedge \rho)\right|^{q} d s\right. \\
& \left.+\left(\Delta^{\frac{q}{2}} h^{q}(\Delta) \vee \Delta^{q(\alpha \wedge \theta \wedge \sigma)}\right)+\int_{0}^{t}\left(\mathbb{E}\left|x(s \wedge \rho-\tau)-x_{\Delta}(s \wedge \rho-\tau)\right|^{2 q}\right)^{\frac{1}{2}} d s\right) .
\end{aligned}
$$

Using Gronwall's inequality gives that

$$
\begin{aligned}
\mathbb{E}\left|e_{\Delta}(t \wedge \rho)\right|^{q} & \\
\leq & C\left(\int_{0}^{t} \sup _{0 \leq l \leq s} \mathbb{E}\left|x(l \wedge \rho)-x_{\Delta}(l \wedge \rho)\right|^{q} d s+\left(\Delta^{\frac{q}{2}} h^{q}(\Delta) \vee \Delta^{q(\alpha \wedge \theta \wedge \sigma)}\right)\right. \\
& \left.+\int_{0}^{t}\left(\sup _{0 \leq l \leq s} \mathbb{E}\left|x(l \wedge \rho-\tau)-x_{\Delta}(l \wedge \rho-\tau)\right|^{2 q}\right)^{\frac{1}{2}} d s\right) .
\end{aligned}
$$

Therefore

$$
\begin{array}{rl}
\sup _{0 \leq l \leq t} & \mathbb{E}\left|e_{\Delta}(l \wedge \rho)\right|^{q} \\
\leq & C\left(\int_{0}^{t} \sup _{0 \leq l \leq s} \mathbb{E}\left|x(l \wedge \rho)-x_{\Delta}(l \wedge \rho)\right|^{q} d s+\left(\Delta^{\frac{q}{2}} h^{q}(\Delta) \vee \Delta^{q(\alpha \wedge \theta \wedge \sigma)}\right)\right. \\
& \left.+\int_{0}^{t}\left(\sup _{0 \leq l \leq s} \mathbb{E}\left|x(l \wedge \rho-\tau)-x_{\Delta}(l \wedge \rho-\tau)\right|^{2 q}\right)^{\frac{1}{2}} d s\right) .
\end{array}
$$

Let $y(t)=x(t)-D(x(t-\tau), r(t))$ and $y_{\Delta}(t)=x_{\Delta}(t)-D\left(\bar{x}_{\Delta}(t-\tau), \bar{r}(t)\right)$. Thus $e_{\Delta}(t)=y(t)-$ $y_{\Delta}(t)$. Then for any $c_{3}, c_{4}, c_{5}>0$, we have

$$
\begin{aligned}
& \left|x(t)-x_{\Delta}(t)\right|^{q} \\
& \quad \leq\left(1+c_{3}\right)^{q-1}\left|y(t)-y_{\Delta}(t)\right|^{q}+\left(\frac{1+c_{3}}{c_{3}}\right)^{q-1}\left|D(x(t-\tau), r(t))-D\left(\bar{x}_{\Delta}(t-\tau), \bar{r}(t)\right)\right|^{q} \\
& \quad \leq\left(1+c_{3}\right)^{q-1}\left|e_{\Delta}(t)\right|^{q}+\left(\frac{\left(1+c_{3}\right)\left(1+c_{4}\right)}{c_{3}}\right)^{q-1}\left|D(x(t-\tau), r(t))-D\left(\bar{x}_{\Delta}(t-\tau), r(t)\right)\right|^{q} \\
& \quad+\left(\frac{\left(1+c_{3}\right)\left(1+c_{4}\right)}{c_{3} c_{4}}\right)^{q-1}\left|D\left(\bar{x}_{\Delta}(t-\tau), r(t)\right)-D\left(\bar{x}_{\Delta}(t-\tau), \bar{r}(t)\right)\right|^{q}
\end{aligned}
$$




$$
\begin{aligned}
\leq & \left(1+c_{3}\right)^{q-1}\left|e_{\Delta}(t)\right|^{q}+\left(\frac{\left(1+c_{3}\right)\left(1+c_{4}\right)}{c_{3}}\right)^{q-1} K_{2}^{q}\left|x(t-\tau)-\bar{x}_{\Delta}(t-\tau)\right|^{q} \\
& +\left(\frac{\left(1+c_{3}\right)\left(1+c_{4}\right)}{c_{3} c_{4}}\right)^{q-1}\left|D\left(\bar{x}_{\Delta}(t-\tau), r(t)\right)-D\left(\bar{x}_{\Delta}(t-\tau), \bar{r}(t)\right)\right|^{q} \\
\leq & \left(1+c_{3}\right)^{q-1}\left|e_{\Delta}(t)\right|^{q}+\left(\frac{\left(1+c_{3}\right)\left(1+c_{4}\right)\left(1+c_{5}\right)}{c_{3}}\right)^{q-1} K_{2}^{q}\left|x(t-\tau)-x_{\Delta}(t-\tau)\right|^{q} \\
& +\left(\frac{\left(1+c_{3}\right)\left(1+c_{4}\right)\left(1+c_{5}\right)}{c_{3} c_{5}}\right)^{q-1} K_{2}^{q}\left|x_{\Delta}(t-\tau)-\bar{x}_{\Delta}(t-\tau)\right|^{q} \\
& +\left(\frac{\left(1+c_{3}\right)\left(1+c_{4}\right)}{c_{3} c_{4}}\right)^{q-1}\left|D\left(\bar{x}_{\Delta}(t-\tau), r(t)\right)-D\left(\bar{x}_{\Delta}(t-\tau), \bar{r}(t)\right)\right|^{q} .
\end{aligned}
$$

Choose $c_{3}$ sufficiently large and choose $c_{4}, c_{5}$ sufficiently small such that $c_{6}:=$ $\left(\frac{\left(1+c_{3}\right)\left(1+c_{4}\right)\left(1+c_{5}\right)}{c_{3}}\right)^{q-1} K_{2}^{q}<1$. Then let $c_{7}=\left(\frac{\left(1+c_{3}\right)\left(1+c_{4}\right)\left(1+c_{5}\right)}{c_{3} c_{5}}\right)^{q-1} K_{2}^{q}$ and $c_{8}=\left(\frac{\left(1+c_{3}\right)\left(1+c_{4}\right)}{c_{3} c_{4}}\right)^{q-1}$.

Hence we derive from (3.47) that

$$
\begin{aligned}
& \sup _{0 \leq s \leq t} \mathbb{E}\left|x(s)-x_{\Delta}(s)\right|^{q} \\
& \leq\left(1+c_{3}\right)^{q-1} \sup _{0 \leq s \leq t} \mathbb{E}\left|e_{\Delta}(s)\right|^{q}+c_{6} \sup _{0 \leq s \leq t} \mathbb{E}\left|x(s-\tau)-x_{\Delta}(s-\tau)\right|^{q} \\
&+c_{7} \sup _{0 \leq s \leq t} \mathbb{E}\left|x_{\Delta}(s-\tau)-\bar{x}_{\Delta}(s-\tau)\right|^{q} \\
&+c_{8} \sup _{0 \leq s \leq t} \mathbb{E}\left|D\left(\bar{x}_{\Delta}(s-\tau), r(s)\right)-D\left(\bar{x}_{\Delta}(s-\tau), \bar{r}(s)\right)\right|^{q} \\
& \leq\left(1+c_{3}\right)^{q-1} \sup _{0 \leq s \leq t} \mathbb{E}\left|e_{\Delta}(s)\right|^{q}+c_{6} \sup _{0 \leq s \leq t} \mathbb{E}\left|x(s)-x_{\Delta}(s)\right|^{q} \\
&+c_{7} \sup _{0 \leq s \leq t} \mathbb{E}\left|x_{\Delta}(s)-\bar{x}_{\Delta}(s)\right|^{q}+c_{6} \sup _{-\tau \leq s \leq 0} \mathbb{E}|\xi(s)-\xi(\lfloor s / \Delta\rfloor \Delta)|^{q} \\
&+C(\Delta+o(\Delta)) \\
& \leq\left(1+c_{3}\right)^{q-1} \sup _{0 \leq s \leq t} \mathbb{E}\left|e_{\Delta}(s)\right|^{q}+c_{6} \sup _{0 \leq s \leq t} \mathbb{E}\left|x(s)-x_{\Delta}(s)\right|^{q} \\
&+C\left(\Delta^{\frac{q}{2}} h^{q}(\Delta)+\Delta^{q \alpha}+o(\Delta)\right) .
\end{aligned}
$$

Therefore

$$
\sup _{0 \leq s \leq t} \mathbb{E}\left|x(s)-x_{\Delta}(s)\right|^{q} \leq \frac{\left(1+c_{3}\right)^{q-1}}{1-c_{6}} \sup _{0 \leq s \leq t} \mathbb{E}\left|e_{\Delta}(s)\right|^{q}+C\left(\Delta^{\frac{q}{2}} h^{q}(\Delta)+\Delta^{q \alpha}+o(\Delta)\right) .
$$

Then we have

$$
\begin{aligned}
\sup _{0 \leq l \leq t} \mathbb{E}\left|x(l \wedge \rho)-x_{\Delta}(l \wedge \rho)\right|^{q} \\
\leq C\left(\int_{0}^{t} \sup _{0 \leq l \leq s} \mathbb{E}\left|x(l \wedge \rho)-x_{\Delta}(l \wedge \rho)\right|^{q} d s+\left(\Delta^{\frac{q}{2}} h^{q}(\Delta) \vee \Delta^{q(\alpha \wedge \theta \wedge \sigma)}\right)\right. \\
\left.\quad+\int_{0}^{t}\left(\sup _{0 \leq l \leq s} \mathbb{E}\left|x(l \wedge \rho-\tau)-x_{\Delta}(l \wedge \rho-\tau)\right|^{2 q}\right)^{\frac{1}{2}} d s\right) .
\end{aligned}
$$


An application of Gronwall's inequality gives that

$$
\begin{aligned}
& \sup _{0 \leq s \leq t} \mathbb{E}\left|x(s \wedge \rho)-x_{\Delta}(s \wedge \rho)\right|^{q} \\
& \quad \leq C\left(\Delta_{f}^{q}+\int_{0}^{t}\left(\sup _{0 \leq l \leq s} \mathbb{E}\left|x(l \wedge \rho-\tau)-x_{\Delta}(l \wedge \rho-\tau)\right|^{2 q}\right)^{\frac{1}{2}} d s\right),
\end{aligned}
$$

where $\Delta_{f}=\Delta^{\frac{1}{2}} h(\Delta) \vee \Delta^{(\alpha \wedge \theta \wedge \sigma)}$. Then we use the same technique as in Lemma 3.9 to get the convergence rate. Define

$$
q_{i}=(\lfloor T / \tau\rfloor+2-i) q 2^{\lfloor T / \tau\rfloor+1-i}, \quad i=1,2, \ldots,\lfloor T / \tau\rfloor+1 .
$$

We find that

$$
2 q_{i+1}<q_{i} \quad \text { and } \quad q_{\lfloor T / \tau\rfloor+1}=q, \quad i=1,2, \ldots,\lfloor T / \tau\rfloor .
$$

Note that $\left|x(s-\tau)-x_{\Delta}(s-\tau)\right|=0$ for $s \in[0, \tau]$. Then we derive that

$$
\sup _{0 \leq s \leq \tau} \mathbb{E}\left|x(s \wedge \rho)-x_{\Delta}(s \wedge \rho)\right|^{q_{1}} \leq C \Delta_{f}^{q_{1}} .
$$

Then by Hölder's inequality we obtain that

$$
\begin{aligned}
& \sup _{0 \leq s \leq 2 \tau} \mathbb{E}\left|x(s \wedge \rho)-x_{\Delta}(s \wedge \rho)\right|^{q_{2}} \\
& \quad \leq C\left(\Delta_{f}^{q_{2}}+\int_{0}^{2 \tau}\left(\mathbb{E}\left|x(s \wedge \rho-\tau)-x_{\Delta}(s \wedge \rho-\tau)\right|^{\left.\left.2 q_{2} \frac{q_{1}}{2 q_{2}}\right)^{\frac{q_{2}}{q_{1}}} d s\right) \leq C \Delta_{f}^{q_{2}} .}\right.\right.
\end{aligned}
$$

By induction we could get the desired result. We complete the proof.

Theorem 3.14 Let Assumptions $2.2,2.3,3.1-3.4$, and 3.6 hold. Let $q \in[2, \bar{q})$ and $p>(\beta+$ $\left.l_{v}+2\right) q$. For any sufficiently small $\Delta \in(0,1]$, assume that

$$
h(\Delta) \geq \varphi\left(\left(\Delta^{\frac{q}{2}} h^{q}(\Delta) \vee \Delta^{q(\alpha \wedge \theta \wedge \sigma)}\right)^{\frac{-1}{p-q}}\right) .
$$

Then for every such small $\Delta$, we have

$$
\mathbb{E}\left|x(T)-x_{\Delta}(T)\right|^{q} \leq C\left(\Delta^{\frac{q}{2}} h^{q}(\Delta) \vee \Delta^{q(\alpha \wedge \theta \wedge \sigma)}\right)
$$

and

$$
\mathbb{E}\left|x(T)-\bar{x}_{\Delta}(T)\right|^{q} \leq C\left(\Delta^{\frac{q}{2}} h^{q}(\Delta) \vee \Delta^{q(\alpha \wedge \theta \wedge \sigma)}\right)
$$

for any $T>0$.

Proof Let $\tau_{L}, \tau_{\Delta, L}$, and $\rho_{\Delta, L}$ be as before. Denote $z_{\Delta}(t)=x(t)-x_{\Delta}(t)$. We write $\rho_{\Delta, L}=\rho$ for simplicity. Obviously,

$$
\mathbb{E}\left|z_{\Delta}(T)\right|^{q}=\mathbb{E}\left(\left|z_{\Delta}(T)\right|^{q} \mathbb{I}_{\{\rho>T\}}\right)+\mathbb{E}\left(\left|z_{\Delta}(T)\right|^{q} \mathbb{I}_{\{\rho \leq T\}}\right) .
$$


Let $\delta>0$ be arbitrary. By Young's inequality we get

$$
u^{q} v=\left(\delta u^{p}\right)^{\frac{q}{p}}\left(\frac{v^{p /(p-q)}}{\delta^{q /(p-q)}}\right)^{\frac{p-q}{p}} \leq \frac{q \delta}{p} u^{p}+\frac{p-q}{p \delta^{q /(p-q)}} v^{p /(p-q)}, \quad \forall u, v>0 .
$$

Hence

$$
\mathbb{E}\left(\left|z_{\Delta}(T)\right|^{q} \mathbb{I}_{\{\rho \leq T\}}\right) \leq \frac{q \delta}{p} \mathbb{E}\left|z_{\Delta}(T)\right|^{p}+\frac{p-q}{p \delta^{q /(p-q)}} \mathbb{P}\{\rho \leq T\} .
$$

Applying Lemmas 3.5 and 3.9 gives that

$$
\mathbb{E}\left|z_{\Delta}(T)\right|^{p} \leq C .
$$

By Lemmas 3.11 and 3.12 we have

$$
\mathbb{P}(\rho \leq T) \leq \mathbb{P}\left(\tau_{L} \leq T\right)+\mathbb{P}\left(\tau_{\Delta, L} \leq T\right) \leq \frac{C}{L^{p}} .
$$

Inserting (3.58) and (3.59) into (3.57) yields that

$$
\mathbb{E}\left(\left|z_{\Delta}(T)\right|^{q} \mathbb{I}_{\{\rho \leq T\}}\right) \leq \frac{C q \delta}{p}+\frac{C(p-q)}{p L^{p} \delta^{q /(p-q)}} .
$$

Choose $\delta=\Delta^{\frac{q}{2}} h^{q}(\Delta) \vee \Delta^{q(\alpha \wedge \theta \wedge \sigma)}$ and $L=\left(\Delta^{\frac{q}{2}} h^{q}(\Delta) \vee \Delta^{q(\alpha \wedge \theta \wedge \sigma)}\right)^{\frac{-1}{p-q}}$. Then we have

$$
\mathbb{E}\left|z_{\Delta}(T)\right|^{q} \leq \mathbb{E}\left|z_{\Delta}(T \wedge \rho)\right|^{q}+C\left(\Delta^{\frac{q}{2}} h^{q}(\Delta) \vee \Delta^{q(\alpha \wedge \theta \wedge \sigma)}\right) .
$$

By condition (3.53) we obtain that

$$
\varphi^{-1}(h(\Delta)) \geq\left(\Delta^{\frac{q}{2}} h^{q}(\Delta) \vee \Delta^{q(\alpha \wedge \theta \wedge \sigma)}\right)^{\frac{-1}{p-q}}=L .
$$

We derive from Lemma 3.13 that

$$
\mathbb{E}\left|z_{\Delta}(T)\right|^{q} \leq C\left(\Delta^{\frac{q}{2}} h^{q}(\Delta) \vee \Delta^{q(\alpha \wedge \theta \wedge \sigma)}\right) .
$$

Hence we get the desired result (3.54). Then combing Lemma 3.10 and (3.54) gives (3.55). We complete the proof.

\section{Stability}

In this section, we investigate the almost sure exponential stability of the partially truncated EM method for neutral stochastic differential delay equations with Markovian switching. In order to achieve this aim, we need to assume that Assumption 3.1 holds on $t \in[0, \infty)$. Let $\tilde{F}(t, 0,0, i)=F(t, 0,0, i)=0$ and $\tilde{G}(t, 0,0, i)=G(t, 0,0, i)=0$ for all $t \in[0, \infty)$ and $i \in \mathbb{S}$, which means $f(t, 0,0, i)=g(t, 0,0, i)=0$.

Assumption 4.1 There exist constants $\Lambda \geq 0$ and $\lambda_{1}, \lambda_{2}, \lambda_{3}, \lambda_{4} \geq 0$ satisfying $\lambda_{1}>\lambda_{2}+$ $\lambda_{3}+\lambda_{4}$ such that

$$
2(x-D(y, i))^{T} \tilde{F}(t, x, y, i)+(1+\Lambda)|\tilde{G}(t, x, y, i)|^{2} \leq-\lambda_{1}|x|^{2}+\lambda_{2}|y|^{2}
$$


and

$$
2(x-D(y, i))^{T} F_{\Delta}(t, x, y, i)+\left(1+\Lambda^{-1}\right)\left|G_{\Delta}(t, x, y, i)\right|^{2} \leq \lambda_{3}|x|^{2}+\lambda_{4}|y|^{2}
$$

for all $t \in[0, \infty), x, y \in \mathbb{R}^{n}$, and $i \in \mathbb{S}$.

Remark 4.2 In fact, there are many functions such that $D(y, i), \tilde{F}(t, x, y, i)$, and $\tilde{G}(t, x, y, i)$ satisfying (4.1) and the corresponding $F_{\Delta}(t, x, y, i)$ and $G_{\Delta}(t, x, y, i)$ satisfying (4.2). The example and proof will be given in Sect. 5 .

In the rest of this paper, we set $\Lambda=0$ and $\Lambda^{-1}\left|G_{\Delta}(t, x, y, i)\right|^{2}=0$ if there is no term $G_{\Delta}(t, x, y, i)$. Also, when the linearly growing term $\tilde{G}(t, x, y, i)$ is absent, we set $\Lambda=\infty$ and $\Lambda|\tilde{G}(t, x, y, i)|^{2}=0$.

By Assumption 4.1 we obtain that

$$
\begin{gathered}
2(x-D(y, i))^{T} f_{\Delta}(t, x, y, i)+\left|g_{\Delta}(t, x, y, i)\right|^{2} \\
\leq-\left(\lambda_{1}-\lambda_{3}\right)|x|^{2}+\left(\lambda_{2}+\lambda_{4}\right)|y|^{2}
\end{gathered}
$$

for all $t \in[0, \infty), x, y \in \mathbb{R}^{n}$, and $i \in \mathbb{S}$.

Theorem 4.3 Let Assumptions 2.3, 3.1, and 4.1 hold on $t \in[0, \infty)$. Then the partially truncated EM numerical solution (2.11) is almost surely exponentially stable. Precisely, let $\lambda>0$ be the unique root of

$$
\left(\lambda_{2}+\lambda_{4}\right) e^{\lambda \tau}+\lambda\left(K_{2}+K_{2}^{2}\right) e^{\lambda \tau}+\left(-\lambda_{1}+\lambda_{3}\right)+\lambda\left(1+K_{2}\right)=0
$$

and let $\varepsilon \in\left(0, \frac{\lambda}{2}\right)$ be arbitrary. Then there exists $a \Delta^{*}>0$ such that for any $\Delta<\Delta^{*}$, we have

$$
\limsup _{k \rightarrow \infty} \frac{1}{k \Delta} \log \left|X_{\Delta}\left(t_{k}\right)\right| \leq-\frac{\lambda}{2}+\varepsilon \quad \text { a.s. }
$$

Proof Define

$$
Y\left(t_{k}, X_{\Delta}\left(t_{k}\right), X_{\Delta}\left(t_{k-M}\right), r_{k}^{\Delta}\right)=X_{\Delta}\left(t_{k}\right)-D\left(X_{\Delta}\left(t_{k-M}\right), r_{k}^{\Delta}\right)
$$

Then (2.11) becomes

$$
\begin{aligned}
& Y\left(t_{k+1}, X_{\Delta}\left(t_{k+1}\right), X_{\Delta}\left(t_{k+1-M}\right), r_{k+1}^{\Delta}\right) \\
& \quad= Y\left(t_{k}, X_{\Delta}\left(t_{k}\right), X_{\Delta}\left(t_{k-M}\right), r_{k}^{\Delta}\right)+f_{\Delta}\left(t_{k}, X_{\Delta}\left(t_{k}\right), X_{\Delta}\left(t_{k-M}\right), r_{k}^{\Delta}\right) \Delta \\
& \quad+g_{\Delta}\left(t_{k}, X_{\Delta}\left(t_{k}\right), X_{\Delta}\left(t_{k-M}\right), r_{k}^{\Delta}\right) \Delta B_{k} .
\end{aligned}
$$

We write $Y_{k}=Y\left(t_{k}, X_{\Delta}\left(t_{k}\right), X_{\Delta}\left(t_{k-M}\right), r_{k}^{\Delta}\right), f_{\Delta, k}=f_{\Delta}\left(t_{k}, X_{\Delta}\left(t_{k}\right), X_{\Delta}\left(t_{k-M}\right), r_{k}^{\Delta}\right)$, and $g_{\Delta, k}=$ $g_{\Delta}\left(t_{k}, X_{\Delta}\left(t_{k}\right), X_{\Delta}\left(t_{k-M}\right), r_{k}^{\Delta}\right)$ for simplicity. Hence we have

$$
\left|Y_{k+1}\right|^{2}=\left|Y_{k}\right|^{2}+\left(2 Y_{k}^{T} f_{\Delta, k}+\left|g_{\Delta, k}\right|^{2}+\left|f_{\Delta, k}\right|^{2} \Delta\right) \Delta+m_{\Delta, k}
$$


where

$$
m_{\Delta, k}=\left|g_{\Delta, k} \Delta B_{k}\right|^{2}-\left|g_{\Delta, k}\right|^{2} \Delta+2 f_{\Delta, k}^{T}\left(g_{\Delta, k} \Delta B_{k}\right)+2 Y_{k}^{T}\left(g_{\Delta, k} \Delta B_{k}\right) .
$$

By (3.2) we have

$$
\left|F_{\Delta}(t, x, y, i)\right|^{2} \leq 18 K_{3}^{2}\left(|x|^{2}+|y|^{2}\right) \quad \text { if }|x| \vee|y| \leq 1
$$

and

$$
\left|F_{\Delta}(t, x, y, i)\right|^{2} \leq h^{2}(\Delta) \leq h^{2}(\Delta)\left(|x|^{2}+|y|^{2}\right) \quad \text { if }|x| \vee|y| \geq 1 .
$$

Thus

$$
\begin{aligned}
\left|f_{\Delta, k}\right|^{2} \Delta & \leq 2\left(20 K_{3}^{2}+h^{2}(\Delta)\right) \Delta\left(\left|X_{\Delta}\left(t_{k}\right)\right|^{2}+\left|X_{\Delta}\left(t_{k-M}\right)\right|^{2}\right) \\
& \leq 2\left(20 K_{3}^{2} \Delta+K_{0}^{2} \Delta^{\frac{1}{2}}\right)\left(\left|X_{\Delta}\left(t_{k}\right)\right|^{2}+\left|X_{\Delta}\left(t_{k-M}\right)\right|^{2}\right) \\
& \leq 2\left(20 K_{3}^{2}+K_{0}^{2}\right) \Delta^{\frac{1}{2}}\left(\left|X_{\Delta}\left(t_{k}\right)\right|^{2}+\left|X_{\Delta}\left(t_{k-M}\right)\right|^{2}\right) .
\end{aligned}
$$

Using (4.3) yields that

$$
\begin{aligned}
\left|Y_{k+1}\right|^{2} \leq & \left|Y_{k}\right|^{2}+\left(-\left(\lambda_{1}-\lambda_{3}\right)\left|X_{\Delta}\left(t_{k}\right)\right|^{2}+\left(\lambda_{2}+\lambda_{4}\right)\left|X_{\Delta}\left(t_{k-M}\right)\right|^{2}\right. \\
& \left.+2\left(20 K_{3}^{2}+K_{0}^{2}\right) \Delta^{\frac{1}{2}}\left(\left|X_{\Delta}\left(t_{k}\right)\right|^{2}+\left|X_{\Delta}\left(t_{k-M}\right)\right|^{2}\right)\right) \Delta+m_{\Delta, k} \\
= & \left|Y_{k}\right|^{2}+\left(-\lambda_{1}+\lambda_{3}+2\left(20 K_{3}^{2}+K_{0}^{2}\right) \Delta^{\frac{1}{2}}\right) \Delta\left|X_{\Delta}\left(t_{k}\right)\right|^{2} \\
& +\left(\lambda_{2}+\lambda_{4}+2\left(20 K_{3}^{2}+K_{0}^{2}\right) \Delta^{\frac{1}{2}}\right) \Delta\left|X_{\Delta}\left(t_{k-M}\right)\right|^{2}+m_{\Delta, k} .
\end{aligned}
$$

Let

$$
\begin{aligned}
& P_{\Delta, 1}=-\lambda_{1}+\lambda_{3}+2\left(20 K_{3}^{2}+K_{0}^{2}\right) \Delta^{\frac{1}{2}}, \\
& P_{\Delta, 2}=\lambda_{2}+\lambda_{4}+2\left(20 K_{3}^{2}+K_{0}^{2}\right) \Delta^{\frac{1}{2}} .
\end{aligned}
$$

Therefore, for any positive constant $J>1$, we derive that

$$
\begin{aligned}
J^{(k+1) \Delta} & \left|Y_{k+1}\right|^{2}-J^{k \Delta}\left|Y_{k}\right|^{2} \\
\leq & J^{(k+1) \Delta} P_{\Delta, 1} \Delta\left|X_{\Delta}\left(t_{k}\right)\right|^{2}+J^{(k+1) \Delta} P_{\Delta, 2} \Delta\left|X_{\Delta}\left(t_{k-M}\right)\right|^{2} \\
& +\left(J^{(k+1) \Delta}-J^{k \Delta}\right)\left|Y_{k}\right|^{2}+J^{(k+1) \Delta} m_{\Delta, k} \\
\leq & J^{(k+1) \Delta} P_{\Delta, 1} \Delta\left|X_{\Delta}\left(t_{k}\right)\right|^{2}+J^{(k+1) \Delta} P_{\Delta, 2} \Delta\left|X_{\Delta}\left(t_{k-M}\right)\right|^{2} \\
& +\left(J^{(k+1) \Delta}-J^{k \Delta}\right) 2\left(\left|X_{\Delta}\left(t_{k}\right)\right|^{2}+\left|X_{\Delta}\left(t_{k-M}\right)\right|^{2}\right)+J^{(k+1) \Delta} m_{\Delta, k} \\
\leq & {\left[2\left(1-J^{-\Delta}\right)+P_{\Delta, 1} \Delta\right] J^{(k+1) \Delta}\left|X_{\Delta}\left(t_{k}\right)\right|^{2} } \\
& +\left[2\left(1-J^{-\Delta}\right)+P_{\Delta, 2} \Delta\right] J^{(k+1) \Delta}\left|X_{\Delta}\left(t_{k-M}\right)\right|^{2}+J^{(k+1) \Delta} m_{\Delta, k},
\end{aligned}
$$


which means that

$$
\begin{aligned}
J^{k \Delta}\left|Y_{k}\right|^{2}-\left|Y_{0}\right|^{2} & \\
\leq & {\left[2\left(1-J^{-\Delta}\right)+P_{\Delta, 1} \Delta\right] \sum_{i=0}^{k-1} J^{(i+1) \Delta}\left|X_{\Delta}\left(t_{i}\right)\right|^{2} } \\
& +\left[2\left(1-J^{-\Delta}\right)+P_{\Delta, 2} \Delta\right] \sum_{i=0}^{k-1} J^{(i+1) \Delta}\left|X_{\Delta}\left(t_{i-M}\right)\right|^{2}+\sum_{i=0}^{k-1} J^{(i+1) \Delta} m_{\Delta, i} .
\end{aligned}
$$

Note that

$$
\begin{aligned}
& \sum_{i=0}^{k-1} J^{(i+1) \Delta}\left|X_{\Delta}\left(t_{i-M}\right)\right|^{2} \\
& \quad=\sum_{i=-M}^{-1} J^{(i+1+M) \Delta}\left|X_{\Delta}\left(t_{i}\right)\right|^{2}+\sum_{i=0}^{k-1} J^{(i+1+M) \Delta}\left|X_{\Delta}\left(t_{i}\right)\right|^{2}-\sum_{i=k-M}^{k-1} J^{(i+1+M) \Delta}\left|X_{\Delta}\left(t_{i}\right)\right|^{2} .
\end{aligned}
$$

Thus

$$
J^{k \Delta}\left|Y_{k}\right|^{2}+\left[2\left(1-J^{-\Delta}\right)+P_{\Delta, 2} \Delta\right] \sum_{i=k-M}^{k-1} J^{(i+1+M) \Delta}\left|X_{\Delta}\left(t_{i}\right)\right|^{2} \leq U_{k}
$$

where

$$
\begin{aligned}
U_{k}= & \left|Y_{0}\right|^{2}+\left(\left[2\left(1-J^{-\Delta}\right)+P_{\Delta, 1} \Delta\right]\right. \\
& \left.+\left[2\left(1-J^{-\Delta}\right)+P_{\Delta, 2} \Delta\right] J^{M \Delta}\right) \sum_{i=0}^{k-1} J^{(i+1) \Delta}\left|X_{\Delta}\left(t_{i}\right)\right|^{2} \\
& +\left[2\left(1-J^{-\Delta}\right)+P_{\Delta, 2} \Delta\right] \sum_{i=-M}^{-1} J^{(i+1+M) \Delta}\left|X_{\Delta}\left(t_{i}\right)\right|^{2}+\sum_{i=0}^{k-1} J^{(i+1) \Delta} m_{\Delta, i} \cdot
\end{aligned}
$$

Let us now introduce the function

$$
\begin{aligned}
Q(J)= & \left(2+P_{\Delta, 2} \Delta\right) J^{(M+1) \Delta}-2 J^{M \Delta}+\left(2+P_{\Delta, 1} \Delta\right) J^{\Delta}-2 \\
= & {\left[\left(\lambda_{2}+\lambda_{4}+2\left(20 K_{3}^{2}+K_{0}^{2}\right) \Delta^{\frac{1}{2}}\right) \Delta+2\right] J^{(M+1) \Delta}-2 J^{M \Delta} } \\
& +\left[\left(-\lambda_{1}+\lambda_{3}+2\left(20 K_{3}^{2}+K_{0}^{2}\right) \Delta^{\frac{1}{2}}\right) \Delta+2\right] J^{\Delta}-2 .
\end{aligned}
$$

Define

$$
\Delta_{1}^{*}=\left(\frac{\lambda_{1}-\lambda_{2}-\lambda_{3}-\lambda_{4}}{4\left(20 K_{3}^{2}+K_{0}^{2}\right)}\right)^{2} .
$$

When $\Delta<\Delta_{1}^{*}$, we can observe that

$$
Q(1)=\left[-\left(\lambda_{1}-\lambda_{2}-\lambda_{3}-\lambda_{4}\right)+4\left(20 K_{3}^{2}+K_{0}^{2}\right) \Delta^{\frac{1}{2}}\right] \Delta<0 .
$$


Moreover, choose $\Delta_{2}^{*}>0$ such that for any $\Delta<\Delta_{2}^{*}$,

$$
2+\left(-\lambda_{1}+\lambda_{3}+2\left(20 K_{3}^{2}+K_{0}^{2}\right) \Delta^{\frac{1}{2}}\right) \Delta>0 .
$$

Hence we can derive that for any $J>1$,

$$
\begin{aligned}
Q^{\prime}(J)= & {\left[2 M\left(J^{\Delta}-1\right)+\left(\left(\lambda_{2}+\lambda_{4}+2\left(20 K_{3}^{2}+K_{0}^{2}\right) \Delta^{\frac{1}{2}}\right)(M+1) \Delta+2\right) J^{\Delta}\right] \Delta J^{M \Delta-1} } \\
& +\left[2+\left(-\lambda_{1}+\lambda_{3}+2\left(20 K_{3}^{2}+K_{0}^{2}\right) \Delta^{\frac{1}{2}}\right) \Delta\right] \Delta J^{\Delta-1}>0 .
\end{aligned}
$$

Therefore there exists a unique constant $J_{\Delta}^{*}>1$ such that

$$
Q\left(J_{\Delta}^{*}\right)=0
$$

for any $\Delta<\Delta_{1}^{*} \wedge \Delta_{2}^{*}$. Choosing $J=J_{\Delta}^{*}$ for any $\Delta<\Delta_{1}^{*} \wedge \Delta_{2}^{*}$ yields that

$$
\begin{aligned}
U_{k}= & \left|Y_{0}\right|^{2}+\left[2\left(1-J^{-\Delta}\right)+P_{\Delta, 2} \Delta\right] \sum_{i=-M}^{-1} J^{(i+1+M) \Delta}\left|X_{\Delta}\left(t_{i}\right)\right|^{2} \\
& +\sum_{i=0}^{k-1} J^{(i+1) \Delta} m_{\Delta, i} .
\end{aligned}
$$

Note that the initial sequence $X_{\Delta}\left(t_{i}\right)<\infty$ for any $i=-M,-M+1, \ldots, 0$ and that $\sum_{i=0}^{k-1} J^{(i+1) \Delta} m_{\Delta, i}$ is a martingale. Applying the discrete-type semimartingale convergence theorem gives that for any $\Delta<\Delta_{1}^{*} \wedge \Delta_{2}^{*}$,

$$
\lim _{k \rightarrow \infty} U_{k}<\infty \quad \text { a.s. }
$$

By (4.12) we obtain that

$$
\begin{aligned}
& \limsup _{k \rightarrow \infty}\left(J_{\Delta}^{* k \Delta}\left|Y_{k}\right|^{2}\right) \\
& \quad \leq \limsup _{k \rightarrow \infty}\left(J_{\Delta}^{* k \Delta}\left|Y_{k}\right|^{2}+\left[2\left(1-J^{-\Delta}\right)+P_{\Delta, 2} \Delta\right] \sum_{i=k-M}^{k-1} J_{\Delta}^{*(i+1+M) \Delta}\left|X_{\Delta}\left(t_{i}\right)\right|^{2}\right) \\
& \leq \lim _{k \rightarrow \infty} U_{k}<\infty \quad \text { a.s. }
\end{aligned}
$$

In addition, for any $c_{0}^{*}>0$,

$$
\begin{aligned}
& \sup _{k \geq 0}\left(J_{\Delta}^{* k \Delta}\left|X_{\Delta}\left(t_{k}\right)\right|^{2}\right) \\
& =\sup _{k \geq 0}\left(J_{\Delta}^{* k \Delta}\left|X_{\Delta}\left(t_{k}\right)-D\left(X_{\Delta}\left(t_{k-M}\right), r_{k}^{\Delta}\right)+D\left(X_{\Delta}\left(t_{k-M}\right), r_{k}^{\Delta}\right)\right|^{2}\right) \\
& \leq\left(1+c_{0}^{*}\right) \sup _{k \geq 0}\left(J_{\Delta}^{* k \Delta}\left|X_{\Delta}\left(t_{k}\right)-D\left(X_{\Delta}\left(t_{k-M}\right), r_{k}^{\Delta}\right)\right|^{2}\right) \\
& \quad+\frac{1+c_{0}^{*}}{c_{0}^{*}} \sup _{k \geq 0}\left(J_{\Delta}^{* k \Delta}\left|D\left(X_{\Delta}\left(t_{k-M}\right), r_{k}^{\Delta}\right)\right|^{2}\right)
\end{aligned}
$$




$$
\begin{aligned}
\leq & \left(1+c_{0}^{*}\right) \sup _{k \geq 0}\left(J_{\Delta}^{* k \Delta}\left|X_{\Delta}\left(t_{k}\right)-D\left(X_{\Delta}\left(t_{k-M}\right), r_{k}^{\Delta}\right)\right|^{2}\right) \\
& +\frac{1+c_{0}^{*}}{c_{0}^{*}} K_{2}^{2}\left[\sup _{-M \leq k \leq 0} J_{\Delta}^{*(k+M) \Delta}\left|X_{\Delta}\left(t_{k}\right)\right|^{2}+\sup _{k \geq 0} J_{\Delta}^{*}(k+M) \Delta\left|X_{\Delta}\left(t_{k}\right)\right|^{2}\right] \\
\leq & \left(1+c_{0}^{*}\right) \sup _{k \geq 0}\left(J_{\Delta}^{* k \Delta}\left|X_{\Delta}\left(t_{k}\right)-D\left(X_{\Delta}\left(t_{k-M}\right), r_{k}^{\Delta}\right)\right|^{2}\right)+\frac{1+c_{0}^{*}}{c_{0}^{*}} K_{2}^{2} J_{\Delta}^{* \tau}\|\xi\|^{2} \\
& +\frac{1+c_{0}^{*}}{c_{0}^{*}} K_{2}^{2} J_{\Delta}^{* \tau} \sup _{k \geq 0}\left(J_{\Delta}^{* k \Delta}\left|X_{\Delta}\left(t_{k}\right)\right|^{2}\right) .
\end{aligned}
$$

Then we take $c_{0}^{*}$ sufficiently large such that $\frac{1+c_{0}^{*}}{c_{0}^{*}} K_{2}^{2} J_{\Delta}^{* \tau}<1$ for any $K_{2} \in(0,1)$. Hence

$$
\sup _{k \geq 0}\left(J_{\Delta}^{* k \Delta}\left|X_{\Delta}\left(t_{k}\right)\right|^{2}\right) \leq c_{1}^{*} \sup _{k \geq 0}\left(J_{\Delta}^{* k \Delta}\left|Y_{k}\right|^{2}\right)+c_{2}^{*}\|\xi\|^{2}
$$

where

$$
c_{1}^{*}=\frac{c_{0}^{*}\left(1+c_{0}^{*}\right)}{c_{0}^{*}-\left(1+c_{0}^{*}\right) K_{2}^{2} J_{\Delta}^{* \tau}}, \quad c_{2}^{*}=\frac{\left(1+c_{0}^{*}\right) K_{2}^{2} J_{\Delta}^{* \tau}}{c_{0}^{*}-\left(1+c_{0}^{*}\right) K_{2}^{2} J_{\Delta}^{* \tau}} .
$$

Therefore

$$
\limsup _{k \rightarrow \infty}\left(J_{\Delta}^{* k \Delta}\left|X_{\Delta}\left(t_{k}\right)\right|^{2}\right)<\infty \quad \text { a.s. }
$$

By (4.13) we get that

$$
\begin{aligned}
& {\left[\lambda_{2}+\lambda_{4}+2\left(20 K_{3}^{2}+K_{0}^{2}\right) \Delta^{\frac{1}{2}}\right] J_{\Delta}^{* \tau}+2 J_{\Delta}^{* \tau}\left(1-J^{-\Delta}\right) \frac{1}{\Delta}} \\
& +\left[-\lambda_{1}+\lambda_{3}+2\left(20 K_{3}^{2}+K_{0}^{2}\right) \Delta^{\frac{1}{2}}\right]+2\left(1-J^{-\Delta}\right) \frac{1}{\Delta}=0 .
\end{aligned}
$$

Choose the constant $\vartheta$ such that $J=e^{\vartheta}$. Hence $1-J^{-\Delta}=1-e^{-\vartheta \Delta}$. Define

$$
\begin{aligned}
\bar{Q}_{\Delta}(\vartheta)= & {\left[\lambda_{2}+\lambda_{4}+2\left(20 K_{3}^{2}+K_{0}^{2}\right) \Delta^{\frac{1}{2}}\right] e^{\vartheta \tau}+2 e^{\vartheta \tau}\left(1-e^{-\vartheta \Delta}\right) \frac{1}{\Delta} } \\
& +\left[-\lambda_{1}+\lambda_{3}+2\left(20 K_{3}^{2}+K_{0}^{2}\right) \Delta^{\frac{1}{2}}\right]+2\left(1-e^{-\vartheta \Delta}\right) \frac{1}{\Delta} .
\end{aligned}
$$

Let $\vartheta_{\Delta}^{*}=\log J_{\Delta}^{*}$. Then we have

$$
\bar{Q}_{\Delta}\left(\vartheta_{\Delta}^{*}\right)=0
$$

Since

$$
\lim _{\Delta \rightarrow 0}\left(1-e^{-\vartheta \Delta}\right) \frac{1}{\Delta}=\vartheta
$$

we derive that

$$
\lim _{\Delta \rightarrow 0} \bar{Q}_{\Delta}(\vartheta)=\left(\lambda_{2}+\lambda_{4}\right) e^{\vartheta \tau}+2 \vartheta e^{\vartheta \tau}+\left(-\lambda_{1}+\lambda_{3}\right)+2 \vartheta .
$$


By the definition of $\lambda$ we get from (4.20) and (4.21) that

$$
\lim _{\Delta \rightarrow 0} \vartheta_{\Delta}^{*}=\lambda
$$

which means that for any $\varepsilon \in\left(0, \frac{\lambda}{2}\right)$, there exists $\Delta_{3}^{*}>0$ such that for any $\Delta<\Delta_{3}^{*}$, we have

$$
\vartheta_{\Delta}^{*}>\lambda-2 \varepsilon
$$

We derive from (4.17) and the definition of $\vartheta_{\Delta}^{*}$ that

$$
\limsup _{k \rightarrow \infty} e^{\vartheta_{\Delta}^{*} k \Delta}\left|X_{\Delta}\left(t_{k}\right)\right|^{2}<\infty
$$

Then for any $\Delta<\Delta_{1}^{*} \wedge \Delta_{2}^{*} \wedge \Delta_{3}^{*}=: \Delta^{*}$, we have

$$
\limsup _{k \rightarrow \infty} \frac{1}{k \Delta} \log \left|X_{\Delta}\left(t_{k}\right)\right| \leq-\frac{\lambda}{2}+\varepsilon \quad \text { a.s. }
$$

which is the desired result. We complete the proof.

\section{Example}

Example 5.1 Consider a nonlinear and nonautonomous neutral stochastic differential delay equations with Markovian switching

$$
\begin{aligned}
& d[x(t)-D(x(t-\tau), r(t))] \\
& \quad=f(t, x(t), x(t-\tau), r(t)) d t+g(t, x(t), x(t-\tau), r(t)) d B(t), \quad t \geq 0,
\end{aligned}
$$

with the initial data $x_{0}$ satisfying Assumption 2.2. Here $B(t)$ is a scalar Brownian motion. Moreover $r$ is a Markovian chain on the state space $\mathbb{S}=\{1,2\}$ with generator

$$
\Gamma=\left(\begin{array}{cc}
-2 & 2 \\
1 & -1
\end{array}\right) \text {. }
$$

In addition, for all $t \in[0,1], x, y \in \mathbb{R}^{1}$, and $i \in \mathbb{S}$, let

$$
\begin{aligned}
& D(y, i)=\left\{\begin{array}{ll}
-\frac{1}{6} y & \text { if } i=1, \\
-\frac{1}{12} y & \text { if } i=2,
\end{array} \quad g(t, x, y, i)= \begin{cases}(t(1-t))^{\frac{1}{3}}|y|^{\frac{3}{2}} & \text { if } i=1, \\
(t(1-t))^{\frac{1}{4}}|y|^{\frac{5}{2}} & \text { if } i=2,\end{cases} \right. \\
& f(t, x, y, i)= \begin{cases}-2 y^{3}+(t(1-t))^{\frac{1}{3}} y-10 x+2 y & \text { if } i=1, \\
-4 y^{5}+(t(1-t))^{\frac{1}{4}} y-20 x+2 y & \text { if } i=2 .\end{cases}
\end{aligned}
$$

We easily see that

$$
\begin{aligned}
& \tilde{F}(t, x, y, i)=\left\{\begin{array}{ll}
(t(1-t))^{\frac{1}{3}} y-10 x+2 y & \text { if } i=1, \\
(t(1-t))^{\frac{1}{4}} y-20 x+2 y & \text { if } i=2,
\end{array} \quad \tilde{G}(t, x, y, i)= \begin{cases}0 & \text { if } i=1, \\
0 & \text { if } i=2,\end{cases} \right. \\
& F(t, x, y, i)=\left\{\begin{array}{ll}
-2 y^{3} & \text { if } i=1, \\
-4 y^{5} & \text { if } i=2,
\end{array} \quad G(t, x, y, i)= \begin{cases}(t(1-t))^{\frac{1}{3}}|y|^{\frac{3}{2}} & \text { if } i=1, \\
(t(1-t))^{\frac{1}{4}}|y|^{\frac{5}{2}} & \text { if } i=2 .\end{cases} \right.
\end{aligned}
$$


Obviously, Assumptions 2.3 and 3.1 hold with $K_{3}=20$ and $\beta=4$. Now we verify Assumptions 3.2-3.4 and 3.6. For Assumption 3.2, we get

$$
\begin{aligned}
(x- & D(y, 1)-\bar{x}+D(\bar{y}, 1))^{T}(F(t, x, y, 1)-F(t, \bar{x}, \bar{y}, 1)) \\
& +\frac{\bar{q}-1}{2}|G(t, x, y, 1)-G(t, \bar{x}, \bar{y}, 1)|^{2} \\
\leq & -2(x-\bar{x})\left(y^{3}-\bar{y}^{3}\right)-\frac{1}{3}(y-\bar{y})\left(y^{3}-\bar{y}^{3}\right)+\left.\frac{\bar{q}-1}{2}|| y\right|^{\frac{3}{2}}-\left.|\bar{y}|^{\frac{3}{2}}\right|^{2} \\
\leq & |x-\bar{x}|^{2}+((\bar{q}-1) \vee 5)\left(1+|y|^{4}+|\bar{y}|^{4}\right)|y-\bar{y}|^{2}, \\
(x- & D(y, 2)-\bar{x}+D(\bar{y}, 2))^{T}(F(t, x, y, 2)-F(t, \bar{x}, \bar{y}, 2)) \\
& +\frac{\bar{q}-1}{2}|G(t, x, y, 2)-G(t, \bar{x}, \bar{y}, 2)|^{2} \\
\leq & -4(x-\bar{x})\left(y^{5}-\bar{y}^{5}\right)-\frac{1}{3}(y-\bar{y})\left(y^{5}-\bar{y}^{5}\right)+\left.\frac{\bar{q}-1}{2}|| y\right|^{\frac{5}{2}}-\left.|\bar{y}|^{\frac{5}{2}}\right|^{2} \\
\leq & 2|x-\bar{x}|^{2}+((\bar{q}-1) \vee 40)\left(1+|y|^{8}+|\bar{y}|^{8}\right)|y-\bar{y}|^{2} .
\end{aligned}
$$

Therefore Assumption 3.2 is satisfied. For Assumption 3.3, we derive that

$$
\begin{aligned}
(x- & D(y, 1))^{T} F(t, x, y, 1)+\frac{\bar{p}-1}{2}|G(t, x, y, 1)|^{2} \\
& \leq-2 x y^{3}-\frac{1}{3} y^{4}+\frac{\bar{p}-1}{2}|y|^{3} \leq\left(1+|x|^{2}\right)+((\bar{p}-1) \vee 12)\left(1+|y|^{4}\right)|y|^{2}, \\
(x- & D(y, 2))^{T} F(t, x, y, 2)+\frac{\bar{p}-1}{2}|G(t, x, y, 2)|^{2} \\
& \leq-4 x y^{5}-\frac{1}{3} y^{6}+\frac{\bar{p}-1}{2}|y|^{5} \leq 2\left(1+|x|^{2}\right)+((\bar{p}-1) \vee 40)\left(1+|y|^{8}\right)|y|^{2} .
\end{aligned}
$$

Hence Assumption 3.3 is satisfied. Moreover, Assumption 3.4 holds with $\theta=\sigma=\frac{1}{3} \wedge \frac{1}{4}=\frac{1}{4}$ for $i \in \mathbb{S}$. To verify Assumption 3.6, we need to consider four cases.

Case 1: If $(|x| \vee|y|) \leq \varphi^{-1}(h(\Delta))$, then we have

$$
\begin{aligned}
(x & -D(y, 1))^{T} F_{\Delta}(t, x, y, 1)+\frac{\bar{p}-1}{2}\left|G_{\Delta}(t, x, y, 1)\right|^{2} \\
& =\left(x+\frac{1}{6} y\right)\left(-2 y^{3}\right)+\left.\left.\frac{\bar{p}-1}{2}\left|(t(1-t))^{\frac{1}{3}}\right| y\right|^{\frac{3}{2}}\right|^{2} \\
& \leq\left(1+|x|^{2}\right)+((\bar{p}-1) \vee 12)\left(1+|y|^{4}\right)|y|^{2}, \\
(x & -D(y, 2))^{T} F_{\Delta}(t, x, y, 2)+\frac{\bar{p}-1}{2}\left|G_{\Delta}(t, x, y, 2)\right|^{2} \\
& =\left(x+\frac{1}{12} y\right)\left(-4 y^{5}\right)+\left.\left.\frac{\bar{p}-1}{2}\left|(t(1-t))^{\frac{1}{4}}\right| y\right|^{\frac{5}{2}}\right|^{2} \\
& \leq 2\left(1+|x|^{2}\right)+((\bar{p}-1) \vee 40)\left(1+|y|^{8}\right)|y|^{2} .
\end{aligned}
$$


Case 2: If $(|x| \wedge|y|)>\varphi^{-1}(h(\Delta))$, then we have

$$
\begin{aligned}
(x & -D(y, 1))^{T} F_{\Delta}(t, x, y, 1)+\frac{\bar{p}-1}{2}\left|G_{\Delta}(t, x, y, 1)\right|^{2} \\
& =\left(x+\frac{1}{6} y\right)\left(-2\left(\varphi^{-1}(h(\Delta)) \frac{y}{|y|}\right)^{3}\right)+\left.\left.\frac{\bar{p}-1}{2}\left|(t(1-t))^{\frac{1}{3}}\right| \varphi^{-1}(h(\Delta)) \frac{y}{|y|}\right|^{\frac{3}{2}}\right|^{2} \\
& \leq-2\left(\frac{\varphi^{-1}(h(\Delta))}{|y|}\right)^{3} x y^{3}-\frac{1}{3}\left(\frac{\varphi^{-1}(h(\Delta))}{|y|}\right)^{3} y^{4}+\frac{\bar{p}-1}{2}\left(\frac{\varphi^{-1}(h(\Delta))}{|y|}\right)^{3}|y|^{3} \\
& \leq\left(1+|x|^{2}\right)+((\bar{p}-1) \vee 12)\left(1+|y|^{4}\right)|y|^{2}, \\
(x & -D(y, 2))^{T} F_{\Delta}(t, x, y, 2)+\frac{\bar{p}-1}{2}\left|G_{\Delta}(t, x, y, 2)\right|^{2} \\
& =\left(x+\frac{1}{12} y\right)\left(-4\left(\varphi^{-1}(h(\Delta)) \frac{y}{|y|}\right)^{5}\right)+\left.\left.\frac{\bar{p}-1}{2}\left|(t(1-t))^{\frac{1}{4}}\right| \varphi^{-1}(h(\Delta)) \frac{y}{|y|}\right|^{\frac{5}{2}}\right|^{2} \\
& \leq-4\left(\frac{\varphi^{-1}(h(\Delta))}{|y|}\right)^{5} x y^{5}-\frac{1}{3}\left(\frac{\varphi^{-1}(h(\Delta))}{|y|}\right)^{5} y^{6}+\frac{\bar{p}-1}{2}\left(\frac{\varphi^{-1}(h(\Delta))}{|y|}\right)^{5}|y|^{5} \\
& \leq 2\left(1+|x|^{2}\right)+((\bar{p}-1) \vee 40)\left(1+|y|^{8}\right)|y|^{2} .
\end{aligned}
$$

Case 3: If $|y|>\varphi^{-1}(h(\Delta))$ and $|x|<\varphi^{-1}(h(\Delta))$, then we derive that

$$
\begin{aligned}
(x & -D(y, 1))^{T} F_{\Delta}(t, x, y, 1)+\frac{\bar{p}-1}{2}\left|G_{\Delta}(t, x, y, 1)\right|^{2} \\
& =\left(x+\frac{1}{6} y\right)\left(-2\left(\varphi^{-1}(h(\Delta)) \frac{y}{|y|}\right)^{3}\right)+\left.\left.\frac{\bar{p}-1}{2}\left|(t(1-t))^{\frac{1}{3}}\right| \varphi^{-1}(h(\Delta)) \frac{y}{|y|}\right|^{\frac{3}{2}}\right|^{2} \\
& \leq\left(1+|x|^{2}\right)+((\bar{p}-1) \vee 12)\left(1+|y|^{4}\right)|y|^{2}, \\
(x & -D(y, 2))^{T} F_{\Delta}(t, x, y, 2)+\frac{\bar{p}-1}{2}\left|G_{\Delta}(t, x, y, 2)\right|^{2} \\
& =\left(x+\frac{1}{12} y\right)\left(-4\left(\varphi^{-1}(h(\Delta)) \frac{y}{|y|}\right)^{5}\right)+\left.\left.\frac{\bar{p}-1}{2}\left|(t(1-t))^{\frac{1}{4}}\right| \varphi^{-1}(h(\Delta)) \frac{y}{|y|}\right|^{\frac{5}{2}}\right|^{2} \\
& \leq 2\left(1+|x|^{2}\right)+((\bar{p}-1) \vee 40)\left(1+|y|^{8}\right)|y|^{2} .
\end{aligned}
$$

Case 4: If $|y|<\varphi^{-1}(h(\Delta))$ and $|x|>\varphi^{-1}(h(\Delta))$, then the proof is similar to the previous case.

Combing the four cases, we get that Assumption 3.6 is satisfied as well. Then we choose $\varphi(\cdot)$ and $h(\cdot)$. We can observe that

$$
\sup _{0 \leq t \leq T} \sup _{|x| \vee|y| \leq w}(|F(t, x, y, i)| \vee|G(t, x, y, i)|) \leq 4 w^{5}, \quad \forall w \geq 1
$$

which means that $\varphi(w)=4 w^{5}$. Let $h(\Delta)=K_{0} \Delta^{-\frac{1}{8}}$. Then by Theorem 3.14, when $\alpha=\frac{1}{4}$, we obtain that

$$
\mathbb{E}\left|x(T)-x_{\Delta}(T)\right|^{2} \leq C \Delta^{\frac{1}{2}} \text { and } \quad \mathbb{E}\left|x(T)-\bar{x}_{\Delta}(T)\right|^{2} \leq C \Delta^{\frac{1}{2}} .
$$

Since the explicit solution of (5.1) cannot be calculated, we regard the partially truncated EM scheme with step size $2^{-14}$ as the true solution in the numerical experiments. Figure 1 


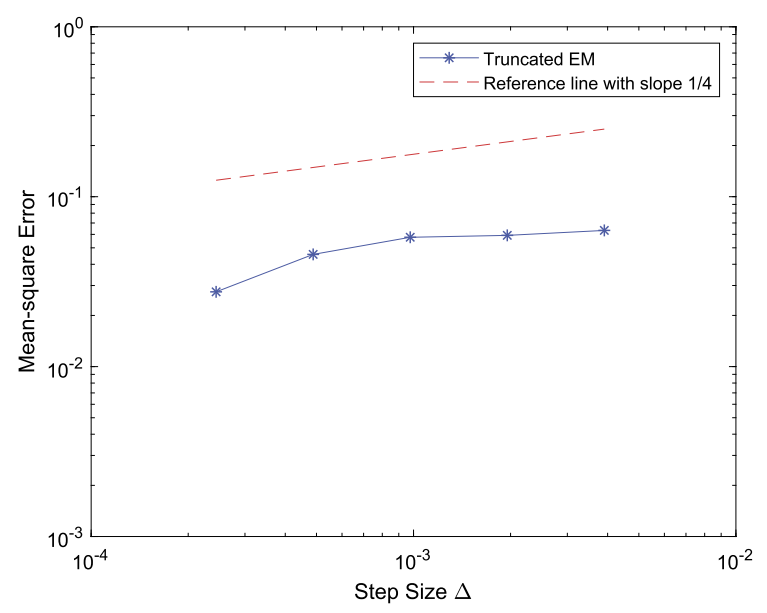

Figure 1 The convergence order of the truncated EM scheme for (5.1)

presents the $\mathscr{L}^{2}$-errors defined by

$$
\left(\mathbb{E}\left|x(T)-x_{\Delta}(T)\right|^{2}\right)^{\frac{1}{2}} \approx\left(\frac{1}{1000} \sum_{i=1}^{1000}\left|(x(T))_{i}-\left(x_{\Delta}(T)\right)_{i}\right|^{2}\right)^{\frac{1}{2}}
$$

with step sizes $2^{-11}, 2^{-10}, 2^{-9}, 2^{-8}, 2^{-7}$ at $T=1.1000$ sample paths were simulated in the numerical experiments. We can observe that the convergence order of partially truncated EM method for (5.1) is approximately $\frac{1}{4}$, which is close to our result.

Example 5.2 Consider a nonlinear and nonautonomous neutral stochastic differential delay equations with Markovian switching

$$
\begin{aligned}
& d[x(t)-D(x(t-\tau), r(t))] \\
& \quad=f(t, x(t), x(t-\tau), r(t)) d t+g(t, x(t), x(t-\tau), r(t)) d B(t), \quad t \geq 0,
\end{aligned}
$$

with the initial data $x_{0}$ satisfying Assumption 2.2. Here $B(t)$ and the Markovian chain are the same as Example 5.1. In addition, for all $t \in[0, \infty), x, y \in \mathbb{R}^{1}$, and $i \in \mathbb{S}$, let

$$
\begin{aligned}
& D(y, i)=\left\{\begin{array}{ll}
\frac{1}{6} \sin y & \text { if } i=1, \\
\frac{1}{12} \sin y & \text { if } i=2,
\end{array} \quad g(t, x, y, i)= \begin{cases}|\sin (t(1-t))|^{\frac{1}{3}}|x|^{\frac{3}{2}} & \text { if } i=1, \\
|\sin (t(1-t))|^{\frac{1}{4}}|x|^{\frac{5}{2}} & \text { if } i=2,\end{cases} \right. \\
& f(t, x, y, i)= \begin{cases}-2 x^{3}+|\sin (t(1-t))|^{\frac{1}{3}} y-10 x+2 y & \text { if } i=1, \\
-4 x^{5}+|\sin (t(1-t))|^{\frac{1}{4}} y-20 x+2 y & \text { if } i=2 .\end{cases}
\end{aligned}
$$

It is easy to see that

$$
\tilde{F}(t, x, y, i)=\left\{\begin{array}{ll}
|\sin (t(1-t))|^{\frac{1}{3}} y-10 x+2 y & \text { if } i=1, \\
|\sin (t(1-t))|^{\frac{1}{4}} y-20 x+2 y & \text { if } i=2,
\end{array} \quad \tilde{G}(t, x, y, i)= \begin{cases}0 & \text { if } i=1, \\
0 & \text { if } i=2,\end{cases}\right.
$$




$$
F(t, x, y, i)=\left\{\begin{array}{ll}
-2 x^{3} & \text { if } i=1, \\
-4 x^{5} & \text { if } i=2,
\end{array} \quad G(t, x, y, i)= \begin{cases}|\sin (t(1-t))|^{\frac{1}{3}}|x|^{\frac{3}{2}} & \text { if } i=1, \\
|\sin (t(1-t))|^{\frac{1}{4}}|x|^{\frac{5}{2}} & \text { if } i=2 .\end{cases}\right.
$$

Obviously, $D(y, i)$ satisfies Assumption 2.3 for $i \in \mathbb{S}$. Now let us check Assumption 4.1. There is no $\tilde{G}(t, x, y, i)$ term, so we have $\Lambda=\infty$. Then we derive that

$$
\begin{aligned}
& 2(x-D(y, 1))^{T} \tilde{F}(t, x, y, 1) \leq-16|x|^{2}+5|y|^{2}, \\
& 2(x-D(y, 2))^{T} \tilde{F}(t, x, y, 2) \leq-35|x|^{2}+6|y|^{2} .
\end{aligned}
$$

Then like in verifying Assumption 3.6, we need to consider the second inequality in four cases.

Case 1: If $(|x| \vee|y|) \leq \varphi^{-1}(h(\Delta))$, then we get

$$
\begin{aligned}
2(x & -D(y, 1))^{T} F_{\Delta}(t, x, y, 1)+\left|G_{\Delta}(t, x, y, 1)\right|^{2} \\
& =2\left(x-\frac{1}{6} \sin y\right)\left(-2 x^{3}\right)+\left.\left.|| \sin (t(1-t))\right|^{\frac{1}{3}}|x|^{\frac{3}{2}}\right|^{2} \\
& \leq-|x|^{2}\left(-2|x|+\frac{1}{2}\right)^{2}+\frac{1}{4}|x|^{2} \leq \frac{1}{4}|x|^{2}+\frac{1}{4}|y|^{2}, \\
2(x & -D(y, 2))^{T} F_{\Delta}(t, x, y, 2)+\left|G_{\Delta}(t, x, y, 2)\right|^{2} \\
& =2\left(x-\frac{1}{12} \sin y\right)\left(-4 x^{5}\right)+\left.\left.|| \sin (t(1-t))\right|^{\frac{1}{4}}|x|^{\frac{5}{2}}\right|^{2} \\
& \leq-2|x|^{2}\left(-2|x|^{2}+\frac{1}{4}\right)^{2}+\frac{1}{8}|x|^{2} \leq \frac{1}{8}|x|^{2}+\frac{1}{8}|y|^{2} .
\end{aligned}
$$

Case 2: If $(|x| \wedge|y|)>\varphi^{-1}(h(\Delta))$, then we have

$$
\begin{aligned}
2(x & -D(y, 1))^{T} F_{\Delta}(t, x, y, 1)+\left|G_{\Delta}(t, x, y, 1)\right|^{2} \\
& =2\left(x-\frac{1}{6} \sin y\right)\left(-2\left(\varphi^{-1}(h(\Delta)) \frac{x}{|x|}\right)^{3}\right)+\left.\left.|| \sin (t(1-t))\right|^{\frac{1}{3}}\left|\varphi^{-1}(h(\Delta)) \frac{x}{|x|}\right|^{\frac{3}{2}}\right|^{2} \\
& \leq-4\left(\frac{\varphi^{-1}(h(\Delta))}{|x|}\right)^{3}|x|^{4}+\frac{2}{3}\left(\frac{\varphi^{-1}(h(\Delta))}{|x|}\right)^{3}|x|^{3}+\left(\frac{\varphi^{-1}(h(\Delta))}{|x|}\right)^{3}|x|^{3} \\
& \leq-\left(\frac{\varphi^{-1}(h(\Delta))}{|x|}\right)^{3}|x|^{2}\left(-2|x|+\frac{1}{2}\right)^{2}+\frac{1}{4}|x|^{2} \leq \frac{1}{4}|x|^{2}+\frac{1}{4}|y|^{2}, \\
2(x & -D(y, 2))^{T} F_{\Delta}(t, x, y, 2)+\left|G_{\Delta}(t, x, y, 2)\right|^{2} \\
& =2\left(x-\frac{1}{12} \sin y\right)\left(-4\left(\varphi^{-1}(h(\Delta)) \frac{x}{|x|}\right)^{5}\right)+\left.\left.|| \sin (t(1-t))\right|^{\frac{1}{4}}\left|\varphi^{-1}(h(\Delta)) \frac{x}{|x|}\right|^{\frac{5}{2}}\right|^{2} \\
& \leq-8\left(\frac{\varphi^{-1}(h(\Delta))}{|x|}\right)^{5} x^{6}+\frac{2}{3}\left(\frac{\varphi^{-1}(h(\Delta))}{|x|}\right)^{5}|x|^{5}+\left(\frac{\varphi^{-1}(h(\Delta))}{|x|}\right)^{5}|x|^{5} \\
& \leq-2\left(\frac{\varphi^{-1}(h(\Delta))}{|x|}\right)^{5}|x|^{2}\left(-2|x|^{2}+\frac{1}{4}\right)^{2}+\frac{1}{8}|x|^{2} \leq \frac{1}{8}|x|^{2}+\frac{1}{8}|y|^{2} .
\end{aligned}
$$




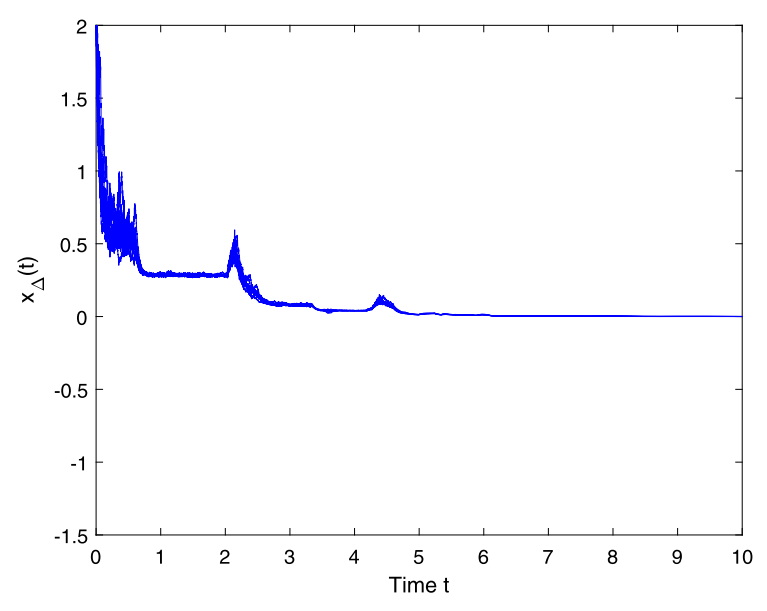

Figure 210 sample paths of $X_{\Delta}(t)$ for (5.2)

Case 3: If $|x|>\varphi^{-1}(h(\Delta))$ and $|y|<\varphi^{-1}(h(\Delta))$, then we derive that

$$
\begin{aligned}
2(x & -D(y, 1))^{T} F_{\Delta}(t, x, y, 1)+\left|G_{\Delta}(t, x, y, 1)\right|^{2} \\
& =2\left(x-\frac{1}{6} \sin y\right)\left(-2\left(\varphi^{-1}(h(\Delta)) \frac{x}{|x|}\right)^{3}\right)+\left.\left.|| \sin (t(1-t))\right|^{\frac{1}{3}}\left|\varphi^{-1}(h(\Delta)) \frac{x}{|x|}\right|^{\frac{3}{2}}\right|^{2} \\
& \leq \frac{1}{4}|x|^{2}+\frac{1}{4}|y|^{2}, \\
2(x-D(y, 2))^{T} F_{\Delta}(t, x, y, 2)+\left|G_{\Delta}(t, x, y, 2)\right|^{2} & \\
& =2\left(x-\frac{1}{12} \sin y\right)\left(-4\left(\varphi^{-1}(h(\Delta)) \frac{x}{|x|}\right)^{5}\right)+\left.\left.|| \sin (t(1-t))\right|^{\frac{1}{4}}\left|\varphi^{-1}(h(\Delta)) \frac{x}{|x|}\right|^{\frac{5}{2}}\right|^{2} \\
& \leq \frac{1}{8}|x|^{2}+\frac{1}{8}|y|^{2} .
\end{aligned}
$$

Case 4: If $|x|<\varphi^{-1}(h(\Delta))$ and $|y|>\varphi^{-1}(h(\Delta))$, then the proof is similar to the above process. Therefore Assumption 4.1 holds. Moreover, we easily to see that Assumption 3.1 is satisfied on $t \in[0, \infty)$. Then by Theorem 4.3 the partially truncated EM numerical solution is almost surely exponentially stable. Figure 2 shows the almost sure exponential stability of the partially truncated EM method for (5.2) with 10 sample paths.

\section{Acknowledgements}

The authors would like to thank the anonymous reviewers for their work and constructive comments, which improved the manuscript.

\section{Funding}

This work is supported by the National Natural Science Foundation of China (Grant Nos. 61876192 and 62076106) and the Fundamental Research Funds for the Central Universities of South-Central University for Nationalities (Grant Nos. KTZ20051, CZT20020, and CZT20022).

Availability of data and materials

Not applicable. 
Authors' contributions

Both authors contributed equally to each part of this work. Both authors read and approved the final manuscript.

\section{Publisher's Note}

Springer Nature remains neutral with regard to jurisdictional claims in published maps and institutional affiliations.

\section{Received: 17 August 2020 Accepted: 10 November 2020 Published online: 08 December 2020}

\section{References}

1. Anderson, D.F., Higham, D.J., Sun, Y.: Multilevel Monte Carlo for stochastic differential equations with small noise. SIAM J. Numer. Anal. 54, 505-529 (2016)

2. Appleby, J.A.D., Guzowska, M., Kelly, C., Rodkina, A.: Preserving positivity in solutions of discretised stochastic differential equations. Appl. Math. Comput. 217, 763-774 (2010)

3. Arnold, L.: Stochastic Differential Equations, Theory and Applications. Wiley, New York (1974)

4. Burrage, K., Tian, T.: Predictor-corrector methods of Runge-Kutta type for stochastic differential equations. SIAM J. Numer. Anal. 40, 1516-1537 (2002)

5. Cong, Y., Zhan, W., Guo, Q.: The partially truncated Euler-Maruyama method for highly nonlinear stochastic delay differential equations with Markovian switching. Int. J. Comput. Methods (2019). https://doi.org/10.1142/S0219876219500142

6. Guo, Q., Liu, W., Mao, X., Yue, R.: The partially truncated Euler-Maruyama method and its stability and boundedness Appl. Numer. Math. 115, 235-251 (2017)

7. Guo, Q., Mao, X., Yue, R.: The truncated Euler-Maruyama method for stochastic differential delay equations. Numer. Algorithms 78, 599-624 (2018)

8. Higham, D.J., Mao, X., Stuart, A.M.: Strong convergence of Euler-type methods for nonlinear stochastic differential equations. SIAM J. Numer. Anal. 40, 1041-1063 (2002)

9. Hutzenthaler, M., Jentzen, A., Kloeden, P.E.: Strong and weak divergence in finite time of Euler's method for stochastic differential equations with non-globally Lipschitz continuous coefficients. Proc. R. Soc. A 467, 1563-1576 (2010)

10. Hutzenthaler, M., Jentzen, A., Kloeden, P.E.: Strong convergence of an explicit numerical method for SDEs with nonglobally Lipschitz continuous coefficients. Ann. Appl. Probab. 22, 1611-1641 (2012)

11. Kloeden, P.E., Platen, E.: Numerical Solution of Stochastic Differential Equations. Springer, Berlin (1992)

12. Kolmanovskii, V., Koroleva, N., Maizenberg, T., Mao, X., Matasov, A.: Neutral stochastic differential delay equation with Markovian switching. Stoch. Anal. Appl. 21, 819-847 (2003)

13. Lan, G.: Asymptotic exponential stability of modified truncated EM method for neutral stochastic differential delay equations. J. Comput. Appl. Math. 340, 334-341 (2018)

14. Lan, G., Yuan, C.: Exponential stability of the exact solutions and $\theta$-EM approximations to neutral SDDEs with Markov switching. J. Comput. Appl. Math. 285, 230-242 (2015)

15. Li, X., Mao, X.: A note on almost sure asymptotic stability of neutral stochastic delay differential equations with Markovian switching. Automatica 48, 2329-2334 (2012)

16. Li, X., Mao, X., Yin, G.: Explicit numerical approximations for stochastic differential equations in finite and infinite horizons: truncation methods, convergence in pth moment and stability. IMA J. Numer. Anal. 39, 847-892 (2019)

17. Liu, W., Foondun, M., Mao, X.: Mean square polynomial stability of numerical solutions to a class of stochastic differential equations. Stat. Probab. Lett. 92, 173-182 (2014)

18. Liu, W., Mao, X.: Strong convergence of the stopped Euler-Maruyama method for nonlinear stochastic differential equations. Appl. Math. Comput. 223, 389-400 (2013)

19. Liu, W., Mao, X., Tang, J., Wu, Y.: Truncated Euler-Maruyama method for classical and time-changed non-autonomous stochastic differential equations. Appl. Numer. Math. 153, 66-81 (2020)

20. Mao, X.: Stochastic Differential Equations and Applications. Horwood, Chichester (2007)

21. Mao, X.: The truncated Euler-Maruyama method for stochastic differential equations. J. Comput. Appl. Math. 290 370-384 (2015)

22. Mao, X.: Convergence rates of the truncated Euler-Maruyama method for stochastic differential equations. J. Comput. Appl. Math. 296, 362-375 (2016)

23. Mao, X., Shen, Y.: Almost surely asymptotic stability of neutral stochastic differential delay equations with Markovian switching. Stoch. Process. Appl. 118, 1385-1406 (2008)

24. Mao, X., Yuan, C.: Stochastic Differential Equations with Markovian Switching. Imperial College Press, London (2006)

25. Mao, X., Yuan, C., Yin, G.: Approximations of Euler-Maruyama type for stochastic differential equations with Markovian switching, under non-Lipschitz conditions. J. Comput. Appl. Math. 205, 936-948 (2007)

26. Milstein, G.N., Platen, E., Schurz, H.: Balanced implicit methods for stiff stochastic system. SIAM J. Numer. Anal. 35 , 1010-1019 (1998)

27. Øksendal, B.: Stochastic Differential Equations: An Introduction with Applications. Springer, Berlin (2000)

28. Sabanis, S.: A note on tamed Euler approximations. Electron. Commun. Probab. 18, 47 (2013)

29. Sabanis, S.: Euler approximations with varying coefficients: the case of superlinearly growing diffusion coefficients. Ann. Appl. Probab. 26, 2083-2105 (2016)

30. Saito, Y., Mitsui, T.: T-stability of numerical scheme for stochastic differential equations. World Sci. Ser. Appl. Anal. 2 333-344 (1993)

31. Skorohod, A.V.: Asymptotic Methods in the Theory of Stochastic Differential Equations. Am. Math. Soc., Providence (1989)

32. Szpruch, L., Mao, X., Higham, D., Pan, J.: Numerical simulation of a strongly nonlinear Ait-Sahalia-type interest rate model. BIT Numer. Math. 51, 405-425 (2011)

33. Tan, L., Yuan, C.: Convergence rates of theta-method for NSDDEs under non-globally Lipschitz continuous coefficients. Bull. Math. Sci. 9, 3231-3243 (2019) 
34. Wu, F., Mao, X.: Numerical solutions of neutral stochastic functional differential equations. SIAM J. Numer. Anal. 46, $1821-1841(2008)$

35. Wu, F., Mao, X., Chen, K.: The Cox-Ingersoll-Ross model with delay and strong convergence of its Euler-Maruyama approximate solutions. Appl. Numer. Math. 59, 2641-2658 (2009)

36. Wu, F., Mao, X., Szpruch, L.: Almost sure exponential stability of numerical solutions for stochastic delay differential equations. Numer. Math. 115, 681-697 (2010)

37. Yuan, C., Glover, W.: Approximate solutions of stochastic differential delay equations with Markovian switching. J. Comput. Appl. Math. 194, 207-226 (2006)

38. Zhang, W., Song, M., Liu, M.: Strong convergence of the partially truncated Euler-Maruyama method for a class of stochastic differential delay equations. J. Comput. Appl. Math. 335, 114-128 (2018)

39. Zhou, S., Wu, F.: Convergence of numerical solutions to neutral stochastic delay differential equations with Markovian switching. J. Comput. Appl. Math. 229, 85-96 (2009)

40. Zong, X., Wu, F., Huang, C.: Exponential mean square stability of the theta approximations for neutral stochastic differential delay equations. J. Comput. Appl. Math. 286, 172-185 (2015)

Submit your manuscript to a SpringerOpen ${ }^{\circ}$ journal and benefit from:

- Convenient online submission

- Rigorous peer review

- Open access: articles freely available online

- High visibility within the field

- Retaining the copyright to your article

Submit your next manuscript at $\boldsymbol{\nabla}$ springeropen.com 